Preprint typeset in JHEP style - PAPER VERSION

KEK-TH-1482

\title{
A practical solution to the sign problem in a matrix model for dynamical compactification
}

\author{
Konstantinos N. Anagnostopoulos ${ }^{a}$, Takehiro Azuma ${ }^{b}$ and Jun Nishimura ${ }^{c, d}$ \\ ${ }^{a}$ Physics Department, National Technical University, \\ Zografou Campus, GR-15780 Athens, Greece \\ ${ }^{b}$ Institute for Fundamental Sciences, Setsunan University, \\ 17-8 Ikeda Nakamachi, Neyagawa, Osaka, 572-8508, Japan \\ ${ }^{c}$ KEK Theory Center, High Energy Accelerator Research Organization, \\ 1-1 Oho, Tsukuba, Ibaraki, 305-0801, Japan \\ ${ }^{d}$ Department of Particle and Nuclear Physics, \\ Graduate University for Advanced Studies (SOKENDAI), \\ 1-1 Oho, Tsukuba, Ibaraki, 305-0801, Japan \\ konstant@mail.ntua.gr, azuma@mpg.setsunan.ac.jp, jnishi@post.kek.jp
}

ABSTRACT: The matrix model formulation of superstring theory offers the possibility to understand the appearance of $4 \mathrm{~d}$ space-time from $10 \mathrm{~d}$ as a consequence of spontaneous breaking of the $\mathrm{SO}(10)$ symmetry. Monte Carlo studies of this issue is technically difficult due to the so-called sign problem. We present a practical solution to this problem generalizing the factorization method proposed originally by two of the authors (K.N.A. and J.N.). Explicit Monte Carlo calculations and large- $N$ extrapolations are performed in a simpler matrix model with similar properties, and reproduce quantitative results obtained previously by the Gaussian expansion method. Our results also confirm that the spontaneous symmetry breaking indeed occurs due to the phase of the fermion determinant, which vanishes for collapsed configurations. We clarify various generic features of this approach, which would be useful in applying it to other statistical systems with the sign problem.

KeYwords: Matrix Models, Nonperturbative Effects, Spontaneous Symmetry Breaking. 


\section{Contents}

1. Introduction 1

2. The model and the sign problem 4

3. Factorization method with a single observable $\quad 8$

3.1 the basic idea $\quad 8$

3.2 practical implementation of the method 9

3.3 asymptotic behaviors of the functions $w_{n}(x) \quad 10$

3.4 results for $\left\langle\tilde{\lambda}_{n}\right\rangle \quad 11$

4. Factorization method with multiple observables $\quad 15$

$\begin{array}{lll}4.1 & \text { the generalized formulation } & 15\end{array}$

$\begin{array}{ll}4.2 \text { analysis for the } \mathrm{SO}(3) \text { symmetric vacuum } & 17\end{array}$

$\begin{array}{ll}4.3 \text { analysis for the } \mathrm{SO}(2) \text { symmetric vacuum } & 19\end{array}$

4.4 estimates on the systematic error $\quad 21$

4.5 comparison with the single-observable analysis 22

4.6 calculation of the free energy 23

4.7 including more observables 25

$\begin{array}{ll}\text { 5. Summary and discussions } & 27\end{array}$

A. Details of the Monte Carlo simulation 28

B. Asymptotic behaviors of the functions $f_{n}^{(0)}(x) \quad 31$

C. Large- $N$ extrapolations in the single-observable analysis 32

D. Large- $N$ extrapolations in the multi-observable analysis $\quad 37$

$\begin{array}{lll}\text { D.1 extrapolations for the } \mathrm{SO}(3) \text { symmetric vacuum } & 37\end{array}$

D.2 extrapolations for the $\mathrm{SO}(2)$ symmetric vacuum 38

\section{Introduction}

One of the biggest puzzles in string theory is that typical space-time dimensionality turns out to be higher than the macroscopically observed four dimensions. As a possible approach to this problem, one may think of space-time as an emergent notion, which arises effectively from matrix degrees of freedom. This idea of "emergent space-time" is nicely realized in the gauge-gravity duality including the famous example of the AdS/CFT correspondence [1]. 
Recently the gauge-gravity duality has been demonstrated from first principles by Monte Carlo simulations of supersymmetric gauge theory in one dimension with 16 supercharges [2-9]. (See ref. [10] for a review.) This theory can be obtained formally by the one-dimensional reduction of ten-dimensional $\mathcal{N}=1 \mathrm{U}(N)$ super Yang-Mills theory, and it provides a low energy description of a stack of $N$ D0 branes in type IIA superstring theory. In particular, in the $N \rightarrow \infty$ limit with large 't Hooft coupling, the one-dimensional gauge theory was conjectured to be dual to the 0-brane solution in type IIA supergravity [11]. Monte Carlo calculations have been performed on the gauge theory side for various quantities such as the internal energy [4-7], the Wilson loop [8] and the two-point correlation functions [9], and the results provided first-principle confirmation of corresponding predictions based on the gauge-gravity duality. One of the most important conclusions [6] is that the black hole thermodynamics of the 0-brane solution has been understood microscopically in terms of open string degrees of freedom attached to the D0 branes, which are described by the gauge theory. In other words the $1 \mathrm{~d} \mathrm{U}(N)$ supersymmetric gauge theory describes the interior structure of the black hole in ten dimensions.

As a closely related but more ambitious conjecture, the IIB matrix model was proposed as a nonperturbative definition of type IIB superstring theory in ten dimensions [12]. This model can be formally obtained by the zero-dimensional reduction of ten-dimensional $\mathcal{N}=$ $1 \mathrm{U}(N)$ super Yang-Mills theory, and it has manifest $\mathrm{SO}(10)$ symmetry. Here the space-time is represented by the eigenvalue distribution of the 10 bosonic matrices [13]. Therefore, the model offers the possibility to realize dynamical compactification, ${ }^{1}$ where the extra dimensions become small due to the spontaneous symmetry breaking (SSB) of SO(10). This scenario has been supported by explicit calculations based on the Gaussian expansion method (GEM). By comparing the free energy of the $\mathrm{SO}(d)$ symmetric vacua with $d=$ $2,4,6,7$, it was shown that $d=4$ is favored at the $3 \mathrm{rd}$ order of the expansion [15]. Higher order calculations confirmed this conclusion [16]. (See refs. [17,18], however.)

Just as Monte Carlo simulations have been useful in studying the gauge-gravity duality from first principles, they are expected to be useful also in addressing the issue of dynamical compactification in the IIB matrix model and many others. Indeed early works on zero-dimensional matrix models [19-23] have provided us with a wealth of information on the large- $N$ limit of the models and the nonperturbative dynamics of their degrees of freedom. When one applies such an approach to the IIB matrix model, one encounters a serious technical problem called the sign problem since the determinant (or Pfaffian, strictly speaking) one obtains from integrating out the fermionic matrices is complex. Due to the fluctuation of the phase, huge cancellations occur in Monte Carlo integration over the bosonic matrices. To overcome this problem, a promising method termed the factorization method has been proposed [24], tested on simple models $[25,26]$ and used in simulations of finite density QCD [27]. (See refs. [28,29] for related works on the QCD phase diagram and refs. [30] for other approaches to the complex action problem.) It resembles previously proposed density of states methods [31] but suggests more general and efficient ways of slicing the configuration space. With the proposed technique it is possible to compute the

\footnotetext{
${ }^{1}$ See ref. [14] for an earlier work suggesting this possibility in the framework of bosonic string field theory.
} 
chosen distribution function in the region which is enhanced due to relatively small fluctuations of the phase. Expectation values can be computed as the easily located minima of the free energy thereby drastically reducing the propagation of errors. It also has the merit of being in principle applicable to a wide range of models and its possible success can have a great impact on various physical problems.

In fact the fluctuations of the phase of the fermion determinant is expected to play a crucial role in the dynamical compactification in the IIB matrix model [32]. ${ }^{2}$ It was shown in ref. [32] that the phase vanishes for collapsed configurations, and that the enhancement due to this property can compensate the entropic suppression for such configurations. In ref. [24] it was shown that the competition between the two effects can make the dominant configurations have very different length scales than in the phase-quenched model suggesting a mechanism for dynamically generating small and large dimensions. Models without the phase factor have also been studied by Monte Carlo simulations, which provided strong evidence that the SSB does not occur in such models [20-22]. These results stress the importance of the role played by the phase factor.

In this paper we study a simple zero-dimensional matrix model [34], which realizes the dynamical compactification. The (non-supersymmetric) model has $\mathrm{SO}(4)$ rotational symmetry and consists of four $N \times N$ bosonic matrices and $N_{f}$ flavors of Weyl fermions in the fundamental representation of $\mathrm{SU}(N)$. The integration over fermions yields a complex determinant. In ref. [34], the large- $N$ limit is taken by keeping the ratio $r=N_{f} / N$ fixed, and it is shown analytically for infinitesimal $r$ that the $\mathrm{SO}(4)$ symmetry is broken down to $\mathrm{SO}(3)$. If the phase is quenched, the $\mathrm{SSB}$ is shown not to occur. For finite $r$, the model has been studied using the GEM [35]. While the exact results for infinitesimal $r$ are consistently reproduced, it is shown that the symmetry actually breaks down to $\mathrm{SO}(2)$ at finite $r$ due to the stronger effect of the phase.

Motivated by these results, we perform Monte Carlo simulation of this simplified model, which is expected to serve as a testing ground for the ideas discussed above concerning the SSB in the IIB matrix model as well as for the method of simulating systems with a complex action. Besides being lower dimensional, the model is much easier to simulate than the IIB matrix model with the computational effort increasing as $N^{3}$ instead of $N^{6}$, since the fermions are in the fundamental representation instead of the adjoint representation. We find that the method with appropriate generalization samples efficiently the configuration space, heavily suppressed in the phase-quenched model but important for the full model, overcoming the so-called overlap problem. The distribution function and the phase of the fermion determinant exhibit nice scaling properties which are important for the extrapolation to large $N$ and to the regions of configuration space in which the phase fluctuation obscures the Monte Carlo measurements. We present detailed analyses of this scaling, and provide its clear understanding based on simple theoretical arguments. Thus we have confirmed that the SSB indeed occurs in this model due to the effect of the phase.

We first apply the factorization method to a single observable as originally formulated in ref. [24]. While the results show that the method works to some extent, we also realize

\footnotetext{
${ }^{2}$ See refs. $[13,33]$ for discussions on other possible mechanisms.
} 
certain discrepancies from the GEM results as well as some puzzles, which we attribute to the remaining overlap problem. In order to solve this problem, we generalize the method to multiple observables, and show that the GEM results can be consistently reproduced. The importance of controlling multiple observables in the factorization method is reported briefly in our previous publication [36].

This paper is organized as follows. In section 2 we review the basic properties of the model, and discuss the sign problem that arises in its Monte Carlo studies. In section 3 we investigate the model by the factorization method with a single observable. In section 4 we generalize the factorization method to multiple observables. Section 5 is devoted to a summary and discussions. In appendix $\mathrm{A}$ we describe the details of the algorithm used in our Monte Carlo simulation. In appendix B we discuss some properties of the functions that play an important role in the method. In appendices $\mathrm{C}$ and $\mathrm{D}$ we present the details of the large- $N$ extrapolations made in our analyses.

\section{The model and the sign problem}

The model we study in this paper is defined by the partition function [34]

$$
\begin{aligned}
Z & =\int d A d \psi d \bar{\psi} \mathrm{e}^{-\left(S_{\mathrm{b}}+S_{\mathrm{f}}\right)}, \\
S_{\mathrm{b}} & =\frac{1}{2} N \operatorname{tr}\left(A_{\mu}\right)^{2}, \\
S_{\mathrm{f}} & =-\bar{\psi}_{\alpha}^{f}\left(\Gamma_{\mu}\right)_{\alpha \beta} A_{\mu} \psi_{\beta}^{f} .
\end{aligned}
$$

The bosonic degrees of freedom are represented by $N \times N$ Hermitian ${ }^{3}$ matrices $A_{\mu}(\mu=$ $1, \cdots, D)$. Hereafter we assume $D$ to be even. ${ }^{4}$ The fermionic degrees of freedom are represented by $\bar{\psi}_{\alpha}^{f}$ and $\psi_{\alpha}^{f}$, which have a spinor index $\alpha=1, \cdots, p$, where $p$ represents the number of components of a $D$-dimensional Weyl spinor

$$
p=2^{D / 2-1} .
$$

They also have a flavor index $f=1, \cdots, N_{\mathrm{f}}$, where $N_{\mathrm{f}}$ represents the number of flavors. The fermionic variables $\bar{\psi}_{\alpha}^{f}$ and $\psi_{\alpha}^{f}$ are $N$-dimensional row and column vectors, respectively, so that the actions (2.2) and (2.3) have an $\mathrm{SU}(N)$ symmetry. The $p \times p$ matrices $\Gamma_{\mu}$ are $\mathrm{SO}(D)$ gamma matrices after the Weyl projection. Thus the actions (2.2) and (2.3) have an $\mathrm{SO}(D)$ symmetry, where the bosonic variables $A_{\mu}$ transform as a vector and the fermionic variables transform as Weyl spinors. The fermionic part can be regarded as the zero volume limit of Weyl fermions interacting with a background gauge field via fundamental coupling.

Integrating out the fermions, we obtain

$$
Z=\int d A \mathrm{e}^{-S_{\mathrm{b}}} Z_{\mathrm{f}}[A]
$$

\footnotetext{
${ }^{3}$ In ref. [35] the matrices $A_{\mu}$ are assumed to be also traceless to simplify the calculations in the Gaussian expansion. In our Monte Carlo studies, we do not impose the tracelessness condition to avoid unnecessary complication, since it is irrelevant in the large- $N$ limit.

${ }^{4}$ For odd $D$, the model can be defined using Dirac fermions instead [34]. In that case, however, the fermion determinant is real, and no $\mathrm{SSB}$ of $\mathrm{SO}(D)$ is expected.
} 
where $Z_{\mathrm{f}}[A]=(\operatorname{det} \mathcal{D})^{N_{\mathrm{f}}}$ and $\mathcal{D}=\Gamma_{\mu} A_{\mu}$ is a $p N \times p N$ matrix. Let us then discuss the properties of the fermion determinant $\operatorname{det} \mathcal{D}$ for a single flavor. First of all, it is complex in general. Under parity transformation $A_{D} \rightarrow-A_{D}, A_{i} \rightarrow A_{i}(i \neq D)$, it transforms as $\operatorname{det} \mathcal{D} \rightarrow(\operatorname{det} \mathcal{D})^{*}$. This implies that $\operatorname{det} \mathcal{D}$ is real ${ }^{5}$ for configurations with $A_{D}=0$. From this fact alone, it follows that the phase of the determinant becomes stationary for configurations with $A_{D}=A_{D-1}=0$ since one cannot have a phase fluctuation within a linear perturbation around such configurations. ${ }^{6}$ These properties of the fermion determinant are analogous to those found in the IIB matrix model [32].

As in the case of the IIB matrix model [32], one can extend the above argument further to arrive at the following statements, which actually play an important role in our analysis. Let us define " $d$-dimensional configurations" $(d \geq 1)$ as such configurations $A_{\mu}$ that can be transformed into $A_{d+1}=\cdots=A_{D}=0$ by an $\mathrm{SO}(D)$ transformation. Then for general $d$-dimensional configurations, we have

$$
\frac{\partial^{k} \Gamma}{\partial A_{\mu_{1}} \cdots \partial A_{\mu_{k}}}=0 \quad \text { for } \quad k=1, \cdots,(D-1)-d,
$$

where $\Gamma$ represents the phase of the fermion determinant $Z_{\mathrm{f}}[A]$.

We take the large- $N$ limit with $r=N_{\mathrm{f}} / N$ fixed, which corresponds to the Veneziano limit. ${ }^{7}$ This is needed to make the fermionic degrees of freedom contribute to the partition function comparably to the bosonic degrees of freedom in the large- $N$ limit. Whether the $\mathrm{SSB}$ of $\mathrm{SO}(D)$ occurs in that limit is the issue we would like to address. For that purpose, we consider the "moment of inertia tensor" $[13,20]$

$$
T_{\mu \nu}=\frac{1}{N} \operatorname{tr}\left(A_{\mu} A_{\nu}\right),
$$

and its real positive eigenvalues $\lambda_{n}(n=1, \cdots, D)$ ordered as

$$
\lambda_{1} \geq \lambda_{2} \geq \cdots \geq \lambda_{D} .
$$

The vacuum expectation values (VEVs) of these eigenvalues $\left\langle\lambda_{n}\right\rangle$ play the role of the order parameters. If they turn out to be unequal in the large- $N$ limit, it implies the SSB of $\mathrm{SO}(D)$. In this model, the sum of the VEV of all the eigenvalues is given exactly as ${ }^{8}$

$$
\sum_{n=1}^{D}\left\langle\lambda_{n}\right\rangle=\left\langle\frac{1}{N} \operatorname{tr}\left(A_{\mu}\right)^{2}\right\rangle=D+p r .
$$

\footnotetext{
${ }^{5}$ In this case, the fermion determinant $Z_{\mathrm{f}}[A]$ for $N_{\mathrm{f}}$ flavors is actually real positive for even $N_{\mathrm{f}}$, although it is not necessarily so for odd $N_{\mathrm{f}}$. There is some numerical evidence, however, that configurations with positive determinant dominate statistically in the large- $N$ limit. Therefore we consider that there is no distinction between odd $N_{\mathrm{f}}$ and even $N_{\mathrm{f}}$ in the large- $N$ limit. In the present work, we always use even $N_{\mathrm{f}}$ to avoid this subtlety.

${ }^{6}$ Obviously the same statements hold for configurations obtained by $\mathrm{SO}(D)$ rotations.

${ }^{7}$ For $r=1$ the fermionic variables can be written in terms of $N \times N$ matrices $\left(\Psi_{\alpha}\right)_{\text {if }}$ and $\left(\bar{\Psi}_{\alpha}\right)_{f i}$. The fermionic part of the action becomes $S_{\mathrm{f}}=-\left(\Gamma_{\mu}\right)_{\alpha \beta} \operatorname{tr}\left(\bar{\Psi}_{\alpha} A_{\mu} \Psi_{\beta}\right)$, which can be compared with a term $S_{\mathrm{f}}=-\left(\Gamma_{\mu}\right)_{\alpha \beta} \operatorname{tr}\left(\bar{\Psi}_{\alpha}\left[A_{\mu}, \Psi_{\beta}\right]\right)$ in the IIB matrix model.

${ }^{8}$ This can be derived by rescaling $A_{\mu}^{\prime}=\kappa^{\frac{1}{2}} A_{\mu}$. Defining $Z(\kappa)=\int d A Z_{\mathrm{f}}[A] \exp \left[-\frac{1}{2} N \operatorname{tr}\left(A_{\mu}^{\prime}\right)^{2}\right]$ and using $Z_{\mathrm{f}}\left[A^{\prime}\right]=\kappa^{p r N^{2} / 2} Z_{\mathrm{f}}[A]$, we obtain $Z(\kappa)=\kappa^{-N^{2}(D+p r) / 2} Z(1)$. Finally $\left\langle\frac{1}{N} \operatorname{tr}\left(A_{\mu}\right)^{2}\right\rangle=-\left.\frac{2}{N^{2}} \frac{\partial}{\partial \kappa} \log Z(\kappa)\right|_{\kappa=1}=$ $D+p r$. The same derivation with the replacement $Z_{\mathrm{f}}[A] \mapsto\left|Z_{\mathrm{f}}[A]\right|$ holds for the phase-quenched model.
} 
From now on, let us consider the $D=4$ case, which implies $p=2$ due to (2.4). The gamma matrices are given by

$$
\Gamma_{1}=\sigma_{1}=\left(\begin{array}{ll}
0 & 1 \\
1 & 0
\end{array}\right), \Gamma_{2}=\sigma_{2}=\left(\begin{array}{cc}
0 & -i \\
i & 0
\end{array}\right), \quad \Gamma_{3}=\sigma_{3}=\left(\begin{array}{cc}
1 & 0 \\
0 & -1
\end{array}\right), \quad \Gamma_{4}=i \sigma_{4}=\left(\begin{array}{ll}
i & 0 \\
0 & i
\end{array}\right) \text {. }
$$

Using a simple technique familiar in random matrix theory, it was shown in the large- $N$ limit and at infinitesimal $r$ that the $\operatorname{VEV}\left\langle\lambda_{n}\right\rangle$ is given as [34]

$$
\left\langle\lambda_{n}\right\rangle=\left\{\begin{array}{l}
1+r+\mathrm{o}(r) \text { for } n=1,2,3, \\
1-r+\mathrm{o}(r) \text { for } n=4,
\end{array}\right.
$$

which implies that the $\mathrm{SO}(4)$ symmetry is broken down to $\mathrm{SO}(3)$. This $\mathrm{SSB}$ is associated with the formation of a condensate $\left\langle\bar{\psi}_{\alpha}^{f} \psi_{\alpha}^{f}\right\rangle$, which is invariant under $\mathrm{SO}(3)$ only.
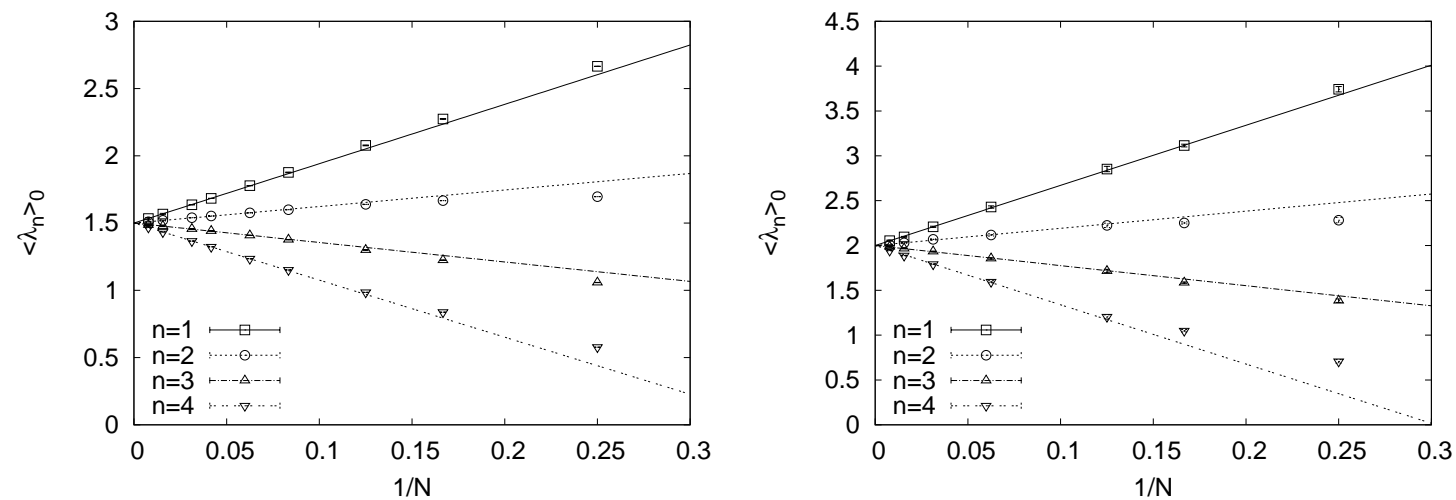

Figure 1: The VEVs $\left\langle\lambda_{n}\right\rangle_{0}(n=1,2,3$ and 4$)$ in the phase-quenched model (2.11) are plotted for $r=1$ (Left) and $r=2$ (Right) against $1 / N$. The data for $N \geq 8$ can be nicely fitted to straight lines meeting at the same point $(1+r / 2)$ at $N=\infty$, which demonstrates the absence of SSB for each $r$.

Here the effect of the phase plays a crucial role. To see that, let us consider the "phase-quenched model"

$$
\begin{aligned}
Z_{0} & =\int d A e^{-S_{0}[A]}, \\
S_{0}[A] & =S_{\mathrm{b}}[A]-N_{\mathrm{f}} \log |\operatorname{det} \mathcal{D}[A]| .
\end{aligned}
$$

Eq. (2.9) holds for this model as well (See footnote 8.), and therefore the absence of SSB would imply in the large- $N$ limit

$$
\left\langle\lambda_{n}\right\rangle_{0}=1+\frac{r}{2} \quad \text { for all } \quad n=1,2,3,4,
$$

where the $\mathrm{VEV}\langle\cdot\rangle_{0}$ is taken with respect to (2.11). Indeed this can be confirmed at infinitesimal $r$ [34]. Here we simulate the system (2.11) for $r=1,2$ and plot the eigenvalues $\left\langle\lambda_{n}\right\rangle_{0}$ against $1 / N$ in fig. 1 . We find that the data can be nicely fitted to straight lines, and the extrapolation to $N=\infty$ gives the value $1+r / 2$ for all $n=1,2,3$ and 4 in accord with $(2.13)^{9}$ with accuracy better than $0.3 \%$. This implies, in particular, that the SSB of

\footnotetext{
${ }^{9}$ Similar results have been obtained for $r=0.25,0.5,1.5,4.0$.
} 
$\mathrm{SO}(4)$ does not occur in the phase-quenched model.

In what follows we will study the normalized eigenvalues

$$
\tilde{\lambda}_{n} \stackrel{\text { def }}{=} \frac{\lambda_{n}}{\left\langle\lambda_{n}\right\rangle_{0}}
$$

The deviation of $\left\langle\tilde{\lambda}_{n}\right\rangle$ from 1 represents the effect of the phase. The relevant question is whether this deviation depends on $n$ in the large- $N$ limit.

\begin{tabular}{|c|c|c|c|c|}
\hline & \multicolumn{2}{|c|}{$r=1$} & \multicolumn{2}{c|}{$r=2$} \\
\hline ansatz & $\mathrm{SO}(3)$ & $\mathrm{SO}(2)$ & $\mathrm{SO}(3)$ & $\mathrm{SO}(2)$ \\
\hline$\left\langle\tilde{\lambda}_{1}\right\rangle$ & 1.17 & 1.4 & 1.23 & 1.7 \\
$\left\langle\tilde{\lambda}_{2}\right\rangle$ & 1.17 & 1.4 & 1.23 & 1.7 \\
$\left\langle\tilde{\lambda}_{3}\right\rangle$ & 1.17 & 0.7 & 1.23 & 0.5 \\
$\left\langle\tilde{\lambda}_{4}\right\rangle$ & 0.5 & 0.5 & 0.31 & 0.1 \\
\hline free energy & -1.5 & -1.8 & -1.9 & -3.6 \\
\hline
\end{tabular}

Table 1: The results for the normalized eigenvalues $\left\langle\tilde{\lambda}_{n}\right\rangle$ and the free energy obtained by the GEM at $N=\infty$ with the $\mathrm{SO}(3)$ and $\mathrm{SO}(2)$ ansatz [35].

At finite $r \leq 2$, the large- $N$ limit of the full model (2.1) was studied by the GEM up to the 9-th order [35] in the same way as it was used in the IIB matrix model $[15,16]$. The results for $r=1$ and $r=2$, which are the cases we focus on in this paper, are summarized in Table 1. We have translated the results for the unnormalized quantities $\left\langle\lambda_{n}\right\rangle$ in ref. [35] to the normalized ones $\left\langle\tilde{\lambda}_{n}\right\rangle$ using (2.13) and (2.14).

The free energy obtained with the $\mathrm{SO}(2)$ ansatz was found to be smaller than the one with the $\mathrm{SO}(3)$ ansatz, which implies that the true vacuum is only $\mathrm{SO}(2)$ symmetric. At smaller $r$ [35], the free energy and the $\operatorname{VEV}\left\langle\lambda_{n}\right\rangle$ obtained with the $\mathrm{SO}(2)$ ansatz asymptotes to the ones obtained with the $\mathrm{SO}(3)$ ansatz, which ensures the consistency with the analytic results (2.10) obtained at infinitesimal $r$.

Monte Carlo simulation of the full model is not straightforward since the complex integrand of (2.5) cannot be regarded as the Boltzmann weight, and hence the idea of importance sampling is not applicable. One approach is to rewrite (2.5) as

$$
Z=\int d A \mathrm{e}^{-S_{0}[A]} \mathrm{e}^{i \Gamma[A]},
$$

and to calculate expectation values by reweighting

$$
\langle\mathcal{O}\rangle=\frac{\left\langle\mathcal{O} \mathrm{e}^{i \Gamma}\right\rangle_{0}}{\left\langle\mathrm{e}^{i \Gamma}\right\rangle_{0}}
$$

where the VEVs on the right-hand side are taken with respect to the phase-quenched model (2.11), and hence can be evaluated, in principle, by standard Monte Carlo techniques. The $\operatorname{VEV}\left\langle\mathrm{e}^{i \Gamma}\right\rangle_{0}$ is nothing but the ratio of the partition functions $Z / Z_{0}$, and it decreases exponentially at large $N$ as $\mathrm{e}^{-N^{2} \Delta F}$, where $\Delta F>0$ is the difference in free energy of the two systems. This can happen due to huge cancellations from the factor $\mathrm{e}^{i \Gamma}$. As a result, one needs $\mathrm{O}\left(\mathrm{e}^{\text {const. }} N^{2}\right)$ configurations to compute an observable with given accuracy by using the formula (2.16) directly. This is called the sign problem.

Another closely related problem is the so-called overlap problem. By using $Z_{0}$ in sampling configurations, we usually sample the wrong region of configuration space, which has little overlap with the dominant configurations in the system given by $Z$. This problem becomes exponentially hard at large $N$ and makes importance sampling inefficient. 


\section{Factorization method with a single observable}

In this section we perform Monte Carlo studies of the model (2.5) by applying the factorization method to a single observable as originally proposed in ref. [24]. While the analysis shows various encouraging behaviors, we will see that the method is only partially successful in reproducing the known results of the GEM. We argue that this is due to the remaining overlap problem, and discuss in section 4 how it can be solved by generalizing the method to multiple observables.

\section{1 the basic idea}

Let us consider calculating the $\operatorname{VEV}\left\langle\tilde{\lambda}_{n}\right\rangle$ by Monte Carlo simulation. Instead of using the reweighting formula (2.16) directly, we first rewrite the VEV as

$$
\left\langle\tilde{\lambda}_{n}\right\rangle=\int_{0}^{\infty} d x x \rho_{n}(x)
$$

in terms of the distribution function

$$
\rho_{n}(x)=\left\langle\delta\left(x-\tilde{\lambda}_{n}\right)\right\rangle .
$$

Applying now the reweighting (2.16) to the right-hand side, one finds that it factorizes as

$$
\rho_{n}(x)=\frac{1}{C} \rho_{n}^{(0)}(x) w_{n}(x)
$$

The real parameter $C$ is a normalization constant given by ${ }^{10}$

$$
C \stackrel{\text { def }}{=}\left\langle e^{i \Gamma}\right\rangle_{0}=\langle\cos \Gamma\rangle_{0}
$$

which need not be calculated in the present method. The real function $\rho_{n}^{(0)}(x)$ is nothing but the distribution function of $\tilde{\lambda}_{n}$ in the phase-quenched model defined by

$$
\rho_{n}^{(0)}(x) \stackrel{\text { def }}{=}\left\langle\delta\left(x-\tilde{\lambda}_{n}\right)\right\rangle_{0},
$$

which is peaked at $x=1$ due to the chosen normalization (2.14). The function $w_{n}(x)$ in (3.3) is defined by

$$
w_{n}(x) \stackrel{\text { def }}{=}\left\langle e^{i \Gamma}\right\rangle_{n, x}=\langle\cos \Gamma\rangle_{n, x}
$$

where $\langle\cdot\rangle_{n, x}$ denotes a VEV with respect to the partition function

$$
Z_{n, x}=\int d A \mathrm{e}^{-S_{0}} \delta\left(x-\tilde{\lambda}_{n}\right)
$$

It turns out that $w_{n}(x)>0$, which simplifies our analysis significantly. ${ }^{11}$

\footnotetext{
${ }^{10}$ In the second equality, we have used the fact that the phase $\Gamma$ flips its sign under the parity transformation $A_{D} \mapsto-A_{D}$, which is a symmetry of the phase-quenched model (2.12). A similar comment applies also to the second equality of (3.6).

${ }^{11}$ This property does not always hold in applications of the factorization method to a system with the complex action problem. See ref. [25] for a study in such a case.
} 
Using the saddle point approximation in the integral (3.1), the problem of determining $\left\langle\tilde{\lambda}_{n}\right\rangle$ can be reduced to that of minimizing the "free energy"

$$
\mathcal{F}_{n}(x)=-\log \rho_{n}(x)
$$

which simply amounts to solving

$$
f_{n}^{(0)}(x) \stackrel{\text { def }}{=} \frac{d}{d x} \log \rho_{n}^{(0)}(x)=-\frac{d}{d x} \log w_{n}(x) .
$$

It turns out that both sides of this equation scales as $\mathrm{O}\left(N^{2}\right)$. Therefore, the saddle point approximation actually becomes exact in the $N \rightarrow \infty$ limit.

\section{2 practical implementation of the method}

As we have discussed above, the calculation of the $\operatorname{VEV}\left\langle\tilde{\lambda}_{n}\right\rangle$ reduces to the determination of the two functions $w_{n}(x)$ and $f_{n}^{(0)}(x)$. Here we discuss how we can measure them by Monte Carlo simulation following ref. [24]. For that purpose we approximate the delta function $\delta\left(x-\tilde{\lambda}_{n}\right)$ in (3.7) by the Gaussian function and simulate the system defined by

$$
Z_{n, V}=\int d A e^{-\left\{S_{0}+V\left(\lambda_{n}\right)\right\}}, \quad V(z)=\frac{1}{2} \gamma(z-\xi)^{2},
$$

where $\gamma$ and $\xi$ are real parameters. Let us then consider the distribution function of $\tilde{\lambda}_{n}$ for the system (3.10), which is given by

$$
\rho_{n, V}(x) \stackrel{\text { def }}{=}\left\langle\delta\left(x-\tilde{\lambda}_{n}\right)\right\rangle_{n, V} \propto \rho_{n}^{(0)}(x) \exp \left\{-V\left(x\left\langle\lambda_{n}\right\rangle_{0}\right)\right\},
$$

where $\langle\cdot\rangle_{n, V}$ represents a VEV with respect to $Z_{n, V}$. The position of the peak, which we denote by $x_{\mathrm{p}}$, can be obtained by solving

$$
0=\frac{d}{d x} \log \rho_{n, V}(x)=f_{n}^{(0)}(x)-\left\langle\lambda_{n}\right\rangle_{0} V^{\prime}\left(x\left\langle\lambda_{n}\right\rangle_{0}\right) .
$$

Since the distribution function $\rho_{n, V}(x)$ is sharply peaked at $x_{\mathrm{p}}$ for sufficiently large $\gamma$, we can use the VEV of $\tilde{\lambda}_{n}$ as an estimator of $x_{\mathrm{p}}$, i.e.,

$$
x_{\mathrm{p}}=\left\langle\tilde{\lambda}_{n}\right\rangle_{n, V} \text {. }
$$

By varying the value of $\xi$ in (3.10), we obtain the functions $w_{n}(x)$ and $f_{n}^{(0)}(x)$ by ${ }^{12}$

$$
\begin{aligned}
w_{n}\left(x_{\mathrm{p}}\right) & =\langle\cos \Gamma\rangle_{n, V}, \\
f_{n}^{(0)}\left(x_{\mathrm{p}}\right) & =\left\langle\lambda_{n}\right\rangle_{0} V^{\prime}\left(\left\langle\lambda_{n}\right\rangle_{n, V}\right)=\gamma\left\langle\lambda_{n}\right\rangle_{0}\left(\left\langle\lambda_{n}\right\rangle_{n, V}-\xi\right) .
\end{aligned}
$$

The parameter $\gamma$ should be chosen large enough to make the fluctuation of $\tilde{\lambda}_{n}$ smaller than the required resolution in $x$. For the purpose of evaluating $f_{n}^{(0)}(x)$, however, one should not

\footnotetext{
${ }^{12}$ One might naively think that the function $\rho_{n}^{(0)}(x)$, and hence the function $f_{n}^{(0)}(x)$, can be obtained by measuring the distribution of $\tilde{\lambda}_{n}$ in the phase-quenched model $Z_{0}$. By such a direct method, however, accurate calculation is possible only in the vicinity of the peak $x=1$. Note that the solution of (3.9) is typically not very close to the peak.
} 
use too large $\gamma$. Theoretically, the right-hand side of (3.15) should converge to the correct value in the $\gamma \rightarrow \infty$ limit, which implies that $\left(\left\langle\lambda_{n}\right\rangle_{n, V}-\xi\right) \propto 1 / \gamma$. Hence a small error in $\left\langle\lambda_{n}\right\rangle_{n, V}$ propagates to $f_{n}^{(0)}(x)$ by the factor of $\gamma$.

Note that the computation of $w_{n}(x)$ based on (3.14) is hard due to the sign problem. However, we only have to obtain $w_{n}(x)$ in the region where the (possibly local) minima of the free energy (3.8) are likely to exist. This typically implies the region where $w_{n}(x)$ is not extremely small. In such a region, one can make a sensible large- $N$ extrapolation

$$
\Phi_{n}(x)=\lim _{N \rightarrow \infty} \frac{1}{N^{2}} \log w_{n}(x) .
$$

Then one can use the equation

$$
\frac{1}{N^{2}} f_{n}^{(0)}(x)=-\frac{d}{d x} \Phi_{n}(x)
$$

to obtain the VEV $\left\langle\tilde{\lambda}_{n}\right\rangle$. Since there is no sign problem in the calculation of $f_{n}^{(0)}(x)$, one may obtain the left-hand side of (3.17) at larger $N$ (if necessary) than the values of $N$ used to obtain $\Phi_{n}(x)$ in eq. (3.16). Note also that the error on both sides of (3.17) due to statistics and finite $N$ does not propagate exponentially to $\left\langle\tilde{\lambda}_{n}\right\rangle$ as a direct computation based on (3.1) would imply.

\section{3 asymptotic behaviors of the functions $w_{n}(x)$}

In our approach the effects of the phase $\Gamma$ are represented by the right-hand side of (3.17). Therefore, the large- $N$ extrapolation (3.16) is the most crucial step for the success of the method. In the left columns of figs. 2 and 3 , we plot our results for $\frac{1}{N^{2}} \log w_{n}(x)$ for $r=1$ and $r=2$, respectively.

As one can see from these plots, the function $w_{n}(x)$ approaches 1 for $x \ll 1$ and/or $x \gg 1$. These are the regions, which are favored by the effects of the phase. We can understand these properties as follows. Let us recall that $w_{n}(x)$ defined by (3.6) is the VEV of $e^{i \Gamma}$ in the ensemble (3.7). The dominant configurations in the ensemble for $x \ll 1$ have $(5-n)$ shrunken directions due to the ordering (2.8), and hence they are approximately configurations with dimension $d=(n-1)$. Similarly, dominant configurations for $x \gg 1$ have $n$ extended directions, and hence they are approximately configurations with dimension $d=n$. Since the phase of the determinant vanishes for collapsed configurations (i.e., with $1 \leq d \leq 3$ dimensions) as we explained below eq. (2.5), the VEV of $e^{i \Gamma}$ approaches 1 when such configurations dominate in the corresponding ensemble.

Moreover, we can deduce the asymptotic behaviors of the functions $w_{n}(x)$ as

$$
\frac{1}{N^{2}} \log w_{n}(x) \simeq \begin{cases}-c_{n} x^{5-n} & (x \ll 1, n=2,3,4), \\ -d_{n} x^{-(4-n)} & (x \gg 1, n=1,2,3) .\end{cases}
$$

This can be derived from the property (2.6) of the phase. It follows that the fluctuation of the phase is of the order of $\delta \Gamma \sim(\delta A /|A|)^{4-d}$ around a collapsed configuration with $1 \leq d \leq 3$ dimensions, where $\delta A$ and $|A|$ represent the typical scale of $A_{\mu}$ in the shrunken 
and extended directions, respectively. Due to the definition (2.7), it is expected that $\delta A /|A|$ is proportional to $\sqrt{x}$ (and $1 / \sqrt{x}$ ) for $x \ll 1$ (and $x \gg 1$ ), respectively. When $x \ll 1$, the distribution of the phase is therefore expected to have a width $\sigma \propto(\sqrt{x})^{5-n}$. Assuming that the distribution is Gaussian, ${ }^{13}$ we can evaluate the expectation value of $e^{i \Gamma}$ by using the formula [28]

$$
\int d \Gamma \frac{1}{\sqrt{2 \pi} \sigma} \exp \left(-\frac{1}{2 \sigma^{2}} \Gamma^{2}\right) e^{i \Gamma}=\exp \left(-\frac{1}{2} \sigma^{2}\right) .
$$

Thus we obtain $-\log w_{n}(x)=\frac{1}{2} \sigma^{2} \propto x^{5-n}$. Similarly, when $x \gg 1$, the distribution is expected to have a width of the order of $(1 / \sqrt{x})^{4-n}$, and hence $-\log w_{n}(x) \propto x^{-(4-n)}$.

We fit our data to the asymptotic behaviors (3.18) and extract the coefficients $c_{n}$ and $d_{n}$ as described in appendix C. It turns out that the finite- $N$ effects in these coefficients are of the order of $1 / N$. Based on this observation, we make large- $N$ extrapolations to obtain (3.16) in the asymptotic regions, which is also plotted in the left columns of figs. 2 and 3. The two solid lines represent the margin of error due to the uncertainty from the large- $N$ extrapolation.

The regions of $x$ in which $\Phi_{n}(x)$ becomes small represent the regions in which the sign problem becomes severe. As we approach such regions, it becomes more difficult to obtain data points, in particular, for larger $N$. A big virtue of the factorization method is that we can use the function $\Phi_{n}(x)$ in the asymptotic regions to extrapolate towards the region where the data points are not available due to the sign problem. One should keep in mind, however, that the true function $\Phi_{n}(x)$ may deviate from the asymptotic behavior as one approaches $x \sim 1$, which causes certain systematic error. We will discuss this issue in sections 4.4 and 4.6 .

\section{4 results for $\left\langle\tilde{\lambda}_{n}\right\rangle$}

On the right columns of figs. 2 and 3 , we plot our results for the function $\frac{1}{N^{2}} f_{n}^{(0)}(x)$ for $r=1$ and $r=2$, respectively. Since there is no sign problem in this calculation, we can obtain results for much larger $N$, which clearly show the large- $N$ scaling behavior. Note also that the function $\frac{1}{N^{2}} f_{n}^{(0)}(x)$ crosses zero at $x \sim 1$ corresponding to the fact that $\rho_{n}^{(0)}(x)$ is peaked at $x \sim 1$. The asymptotic behaviors of the functions $f_{n}^{(0)}(x)$ in the regions $x \ll 1$ and $x \gg 1$ are discussed in appendix B. In the same figures, we also plot $-\frac{d}{d x} \Phi_{n}(x)$ using the scaling function $\Phi_{n}(x)$ obtained as described above. Then the solutions to (3.17) can be obtained as the $x$ coordinates of the intersection points. ${ }^{14}$

For $n=1,4$ there is only one intersection. The effect of the phase is to suppress the $x \sim 1$ region and to shift the corresponding scale to larger/smaller scales, respectively. For $n=2,3$ there are two intersections, which correspond to the local maxima of $\rho_{n}(x)$. The effect of the phase is to produce a double peak structure in $\rho_{n}(x)$, which corresponds to two dynamically generated length scales for the corresponding dimensions. In Table 2 we present the solutions of (3.17) for each $n$, where $x_{\mathrm{s}}$ and $x_{1}$ denotes the solutions in the $x<1$ and $x>1$ regions, respectively.

\footnotetext{
${ }^{13}$ We have checked that this is indeed the case in the region of $x$ where $w_{n}(x)$ is close to 1 .

${ }^{14}$ We will discuss in section 4.5 that in fact there should be more intersecting points that are not seen in these plots. The statements given below ignore this subtle issue.
} 

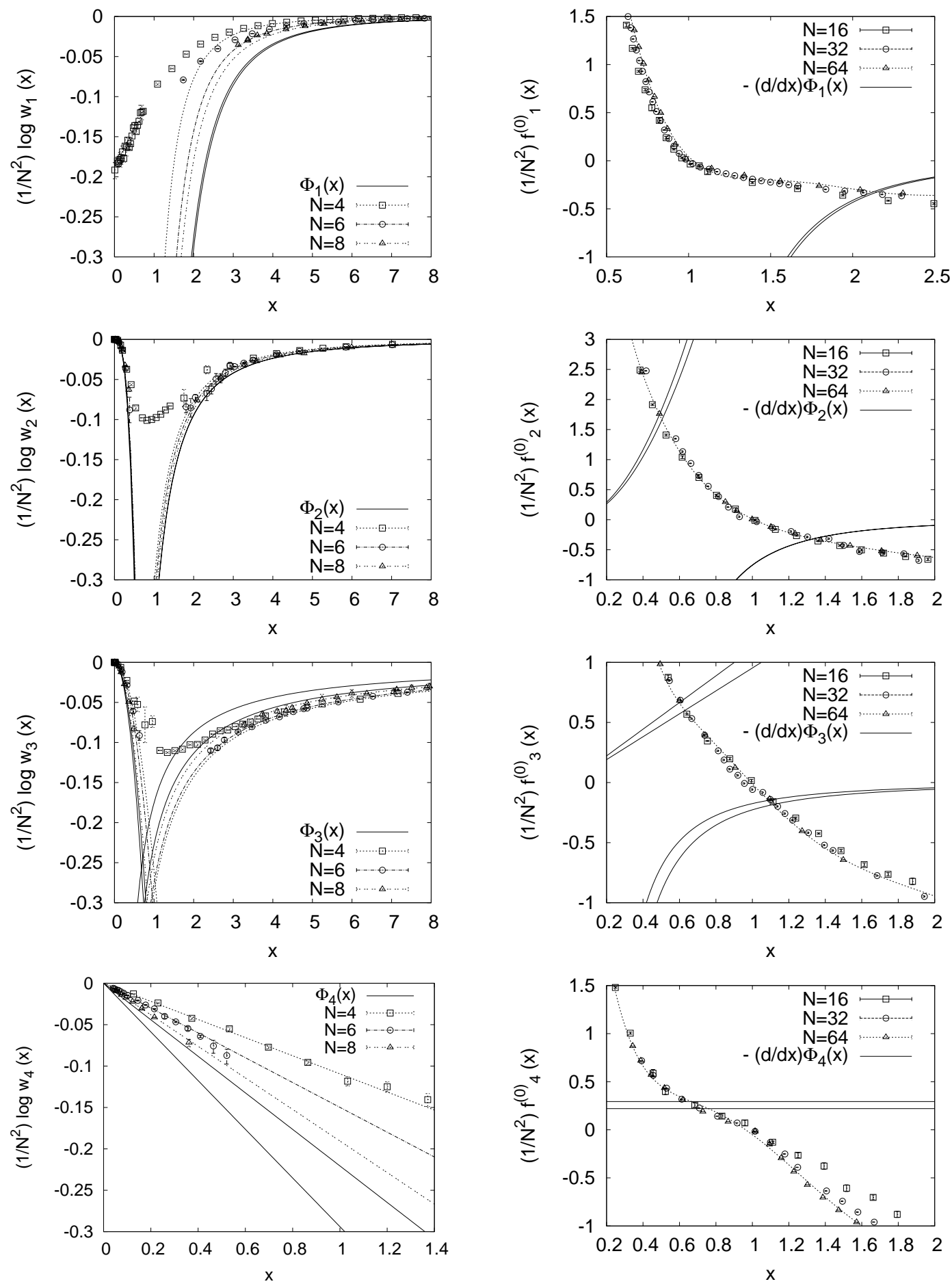

Figure 2: Results for the $r=1$ case. (Left) The function $\frac{1}{N^{2}} \log w_{n}(x)$ is plotted for $N=$ 4, 6, 8 together with the scaling function $\Phi_{n}(x)$ obtained by large- $N$ extrapolations based on the asymptotic behavior (3.18). (Right) The function $\frac{1}{N^{2}} f_{n}^{(0)}(x)$ is plotted for $N=16,32,64$ together with $-\frac{d}{d x} \Phi_{n}(x)$. The $x$ coordinate of the intersecting point gives a solution to (3.17). 

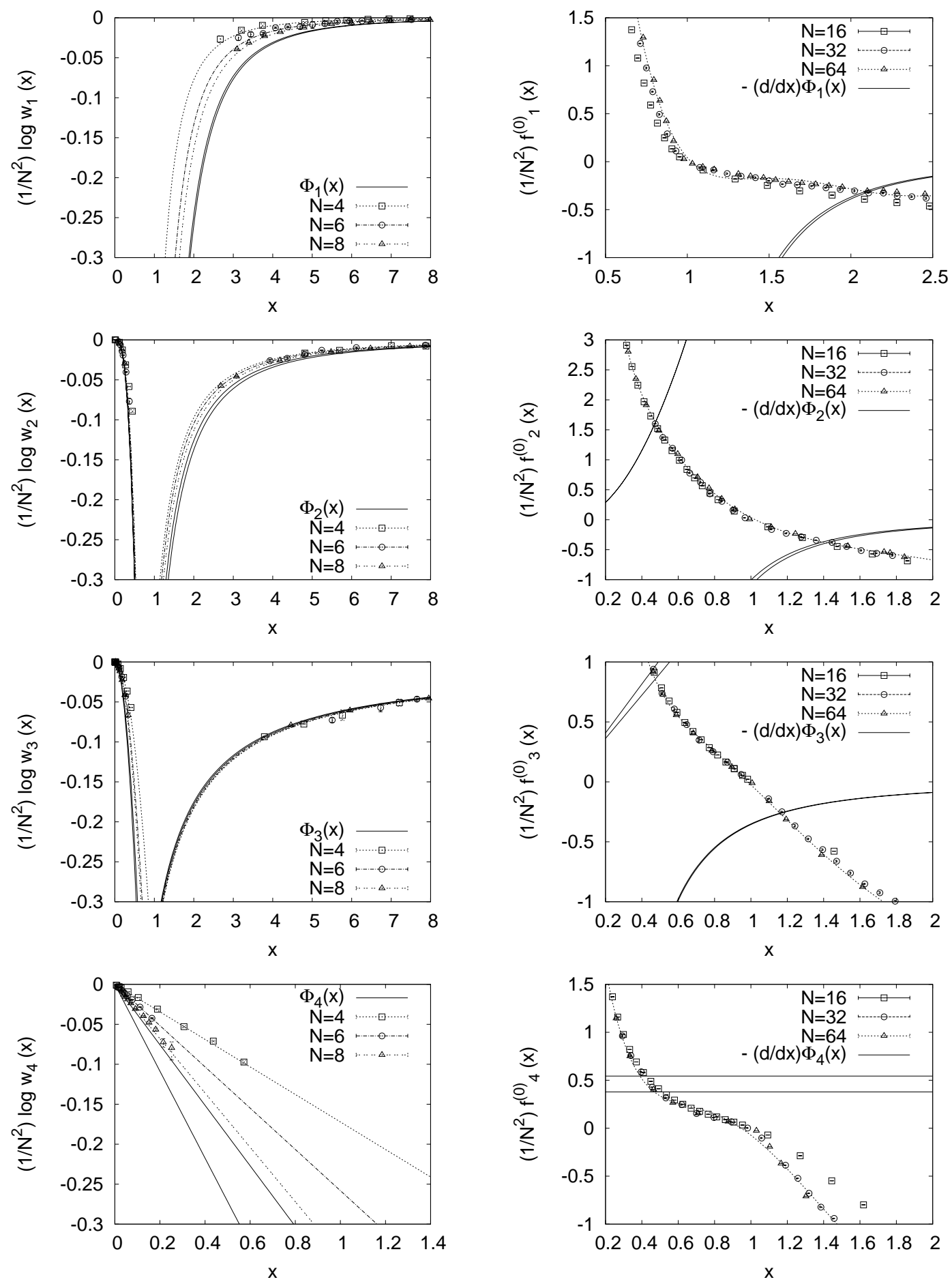

Figure 3: Results for the $r=2$ case. (Left) The function $\frac{1}{N^{2}} \log w_{n}(x)$ is plotted for $N=$ 4, 6, 8 together with the scaling function $\Phi_{n}(x)$ obtained by large- $N$ extrapolations based on the asymptotic behavior (3.18). (Right) The function $\frac{1}{N^{2}} f_{n}^{(0)}(x)$ is plotted for $N=16,32,64$ together with $-\frac{d}{d x} \Phi_{n}(x)$. The $x$ coordinate of the intersecting point gives a solution to (3.17). 


\begin{tabular}{|c|c|c|c|c|c|c|}
\hline & \multicolumn{6}{|c|}{$r=1$} \\
\hline$n$ & $x_{\mathrm{s}}$ & $x_{1}$ & $\Phi_{n}\left(x_{\mathrm{s}}\right)$ & $\Phi_{n}\left(x_{1}\right)$ & $\Xi_{n}$ & $\Delta_{n}$ \\
\hline 1 & & $2.14(1)$ & & $-0.231(3)$ & & \\
2 & $0.49(1)$ & $1.317(1)$ & $-0.274(3)$ & $-0.218(1)$ & $0.27(1)$ & $0.33(2)$ \\
3 & $0.62(2)$ & $1.11(2)$ & $-0.20(1)$ & $-0.18(2)$ & $0.10(2)$ & $0.11(4)$ \\
4 & $0.71(5)$ & & $-0.20(2)$ & & & \\
\hline \hline & \multicolumn{7}{|c|}{$r=2$} \\
\hline$n$ & $x_{\mathrm{s}}$ & $x_{1}$ & $\Phi_{n}\left(x_{\mathrm{S}}\right)$ & $\Phi_{n}\left(x_{1}\right)$ & $\Xi_{n}$ & $\Delta_{n}$ \\
\hline 1 & & $2.12(2)$ & & $-0.213(2)$ & & \\
2 & $0.4718(1)$ & $1.39(1)$ & $-0.2528(1)$ & $-0.27(1)$ & $0.253(5)$ & $0.23(1)$ \\
3 & $0.47(1)$ & $1.159(1)$ & $-0.208(3)$ & $-0.305(2)$ & $0.18(1)$ & $0.08(2)$ \\
4 & $0.44(4)$ & & $-0.20(2)$ & & & \\
\hline
\end{tabular}

Table 2: The first two columns show the solutions $\left(x_{\mathrm{s}}\right.$ and $\left.x_{1}\right)$ to eq. (3.17) that correspond to the (local) maxima of $\rho_{n}(x)$. The other columns show the results for $\Phi_{n}\left(x_{\mathrm{s}}\right), \Phi_{n}\left(x_{1}\right), \Xi_{n}$ and $\Delta_{n}$ that appear in eq. (3.20).

For $n=2,3$, we have to determine which of the two peaks of $\rho_{n}(x)$ actually dominates in the $N \rightarrow \infty$ limit. For that purpose we calculate the difference of the free energy [24]

$$
\begin{aligned}
& \Delta_{n} \stackrel{\text { def }}{=} \frac{1}{N^{2}}\left\{\log \rho_{n}\left(x_{1}\right)-\log \rho_{n}\left(x_{\mathrm{s}}\right)\right\}=\left\{\Phi_{n}\left(x_{1}\right)-\Phi_{n}\left(x_{\mathrm{s}}\right)\right\}+\Xi_{n}, \\
& \text { where } \quad \Xi_{n} \stackrel{\text { def }}{=} \int_{x_{\mathrm{s}}}^{x_{1}} d x\left\{\frac{1}{N^{2}} f_{n}^{(0)}(x)\right\} .
\end{aligned}
$$

If we find that $\Delta_{n}$ is positive (negative), the peak at $x=x_{1}\left(x=x_{\mathrm{s}}\right)$ dominates in the large- $N$ limit. Note that $\Delta_{n}$ depends on the values of $\Phi_{n}(x)$ at $x_{\mathrm{s}}$ and $x_{1}$, but there is no need to know the correct values of $\Phi_{n}(x)$ in the region where we have a severe sign problem. The integral $\Xi_{n}$ can be computed by fitting the data points for largest $N$ to some known function with free parameters in the region $x_{\mathrm{s}} \leq x \leq x_{1}$, and then integrating that function over the region. In Table 2 we also present the results for $\Phi_{n}\left(x_{\mathrm{s}}\right), \Phi_{n}\left(x_{1}\right), \Xi_{n}$ and $\Delta_{n}$. For $n=2$ we obtain a clearly positive value for $\Delta_{n}$, which means that the peak at $x=x_{1}$ dominates. For $n=3$ the obtained value for $\Delta_{n}$ is too small to reliably determine the dominant peak considering the expected systematic errors (See section 4.6.) in $\Phi_{n}\left(x_{\mathrm{s}}\right)$ and $\Phi_{n}\left(x_{1}\right)$.

The results presented above suggest that $\left\langle\tilde{\lambda}_{n}\right\rangle$ indeed deviate from 1 representing the strong effect of the phase, and that we are likely to get $\left\langle\tilde{\lambda}_{1}\right\rangle,\left\langle\tilde{\lambda}_{2}\right\rangle>1>\left\langle\tilde{\lambda}_{4}\right\rangle$, which is consistent with the GEM results. Thus our results support the speculation that the SSB of $\mathrm{SO}(4)$ symmetry occurs due to the effect of the phase. Moreover, the value of $x_{1}$ for $n=2$ and the value of $x_{\mathrm{s}}$ for $n=3$ are reasonably close to the GEM results for $\left\langle\tilde{\lambda}_{2}\right\rangle$ and $\left\langle\tilde{\lambda}_{3}\right\rangle$, respectively, obtained with the $\mathrm{SO}(2)$ ansatz. (See Table 1.)

On the other hand, our results for $\left\langle\tilde{\lambda}_{1}\right\rangle$ and $\left\langle\tilde{\lambda}_{4}\right\rangle$ are not very close to the corresponding GEM results with the $\mathrm{SO}(2)$ ansatz. We also notice a puzzle. In usual Monte Carlo calculations, one obtains various observables using the same set of configurations. However, in the present method, one has to perform independent simulations to obtain $\left\langle\tilde{\lambda}_{n}\right\rangle$ for 
different $n$. In fact the dominant configurations one obtains by simulating the constrained system (3.7) with the parameter $x$ fixed at the GEM results for $\left\langle\tilde{\lambda}_{n}\right\rangle$ can be quite different for different $n$. While this is not a problem per se at least theoretically, it suggests the existence of a practical problem, which is closely related to the observed discrepancies.

For instance, the dominant configurations one obtains from the constrained system (3.7) with $n=1$ and $x>1$ have $\tilde{\lambda}_{1}(=x)>1>\tilde{\lambda}_{2}, \tilde{\lambda}_{3}, \tilde{\lambda}_{4}$. Note that $w_{1}(x)$ is calculated as the VEV of $e^{i \Gamma}$ in such an ensemble. When $x$ is close to $\left\langle\tilde{\lambda}_{1}\right\rangle$ obtained by the GEM with the $\mathrm{SO}(2)$ ansatz, the dominant contribution to $w_{1}(x)$ is expected to come from $\mathrm{SO}(2)$ symmetric configurations due to the enhancement by $e^{i \Gamma}$. This clearly suggests that the overlap problem still remains. As a result, it is expected that $w_{1}(x)$ is underestimated in that region of $x$, which naturally explains the discrepancy for $\left\langle\tilde{\lambda}_{1}\right\rangle$ observed above. Due to this remaining overlap problem, we need to be careful in interpreting the results of the single-observable analysis. We will come back to this point in section 4.5.

\section{Factorization method with multiple observables}

The important point of the method described in the previous section was to consider the distribution function (3.2) instead of trying to calculate the VEV directly by reweighting (2.16). Then the effect of the phase was factorized and represented nicely by the weight function $w_{n}(x)$ defined by (3.6). This function suppresses the region near $x=1$, which is preferred by the phase-quenched model, and enhances the regions $x \ll 1$ and/or $x \gg 1$. By solving the equation (3.17), we can obtain solutions quite different from $x=1$. If we simulate the phase-quenched model, the observables $\tilde{\lambda}_{n}$ fluctuate around $\tilde{\lambda}_{n}=1$, and it would be difficult to sample configurations having $\tilde{\lambda}_{n}$ different from 1 , which represents the overlap problem. By simulating the constrained system (3.7), we are able to estimate the effect of the phase in the region strongly suppressed in the phase-quenched model, and thus the overlap problem can be reduced.

However, we also noticed that the overlap problem can still remain when one evaluates $w_{n}(x)$ defined by eq. (3.6) since it is calculated as the $\mathrm{VEV}$ of $e^{i \Gamma}$ in the constrained system (3.7). There might be some region of the configuration space which cannot be sampled efficiently by simulating the constrained system (3.7), and yet has important contribution to the VEV due to less fluctuation of $\Gamma$. In this section we discuss how we can solve this problem by generalizing the factorization method to multiple observables. Instead of considering the distribution function of $\tilde{\lambda}_{n}$ for each $n$ separately, we consider the distribution function specifying all the $\tilde{\lambda}_{n}(n=1,2,3,4)$ at the same time. We discuss the possibility of including more observables in section 4.7 .

\section{1 the generalized formulation}

We can actually generalize all the formulae in section 3.1 to the multi-observable case in a straightforward manner. The relevant functions for the single-observable case can then be written in terms of those for the multi-observable case. In this generalized formulation, one can clearly identify the overlap problem that still remains in the single-observable analysis. 
Let us begin by defining the distribution functions for multiple observables as

$$
\begin{aligned}
\rho\left(x_{1}, x_{2}, x_{3}, x_{4}\right) & =\left\langle\prod_{k} \delta\left(x_{k}-\tilde{\lambda}_{k}\right)\right\rangle, \\
\rho^{(0)}\left(x_{1}, x_{2}, x_{3}, x_{4}\right) & =\left\langle\prod_{k} \delta\left(x_{k}-\tilde{\lambda}_{k}\right)\right\rangle_{0}
\end{aligned}
$$

for the full model and the phase-quenched model, respectively. By definition (2.8), these functions vanish unless $x_{1} \geq x_{2} \geq x_{3} \geq x_{4}$. Applying the reweighting (2.16) to the righthand side of (4.1), one finds that it factorizes as

$$
\rho\left(x_{1}, x_{2}, x_{3}, x_{4}\right)=\frac{1}{C} \rho^{(0)}\left(x_{1}, x_{2}, x_{3}, x_{4}\right) w\left(x_{1}, x_{2}, x_{3}, x_{4}\right),
$$

where $C$ is a normalization constant given by eq. (3.4). The correction factor $w\left(x_{1}, x_{2}, x_{3}, x_{4}\right)$ is defined by

$$
w\left(x_{1}, x_{2}, x_{3}, x_{4}\right) \stackrel{\text { def }}{=}\left\langle e^{i \Gamma}\right\rangle_{x_{1}, x_{2}, x_{3}, x_{4}}=\langle\cos \Gamma\rangle_{x_{1}, x_{2}, x_{3}, x_{4}},
$$

where $\langle\cdot\rangle_{x_{1}, x_{2}, x_{3}, x_{4}}$ denotes a VEV with respect to the partition function

$$
Z_{x_{1}, x_{2}, x_{3}, x_{4}}=\int d A \mathrm{e}^{-S_{0}} \prod_{k} \delta\left(x_{k}-\tilde{\lambda}_{k}\right) .
$$

Note that all the four observables $\tilde{\lambda}_{n}(n=1,2,3,4)$ are constrained here in contrast to (3.7).

We can rewrite the relevant functions in the single-observable case in terms of the functions defined above. For instance, we have

$$
\begin{aligned}
\rho_{n}\left(x_{n}\right) & =\int \prod_{k \neq n} d x_{k} \rho\left(x_{1}, x_{2}, x_{3}, x_{4}\right), \\
\rho_{n}^{(0)}\left(x_{n}\right) & =\int \prod_{k \neq n} d x_{k} \rho^{(0)}\left(x_{1}, x_{2}, x_{3}, x_{4}\right) .
\end{aligned}
$$

Using (3.3), we also obtain

$$
w_{n}\left(x_{n}\right)=\frac{C \rho_{n}\left(x_{n}\right)}{\rho_{n}^{(0)}\left(x_{n}\right)}=\frac{\int \prod_{k \neq n} d x_{k} \rho^{(0)}\left(x_{1}, x_{2}, x_{3}, x_{4}\right) w\left(x_{1}, x_{2}, x_{3}, x_{4}\right)}{\int \prod_{k \neq n} d x_{k} \rho^{(0)}\left(x_{1}, x_{2}, x_{3}, x_{4}\right)},
$$

where we have used (4.3), (4.6) and (4.7) in the second equality. This formula reveals the possibility that the overlap problem can still occur when one calculates the function $w_{n}(x)$ defined by (3.6) as the VEV of $e^{i \Gamma}$ in the system $Z_{n, x}$. The region that gives dominant contribution in the numerator of (4.8) may not be sampled efficiently by simulating the system $Z_{n, x}$. It is also clear that the overlap problem can be reduced further by constraining all the four observables $\tilde{\lambda}_{n}(n=1,2,3,4)$ at the same time.

Now we have to maximize the distribution function $\rho\left(x_{1}, x_{2}, x_{3}, x_{4}\right)$ with respect to $x_{1}$, $x_{2}, x_{3}$ and $x_{4}$. This leads to the coupled equations

$$
\frac{\partial}{\partial x_{n}} \log \rho^{(0)}\left(x_{1}, x_{2}, x_{3}, x_{4}\right)=-\frac{\partial}{\partial x_{n}} \log w\left(x_{1}, x_{2}, x_{3}, x_{4}\right) \quad \text { for } n=1,2,3,4 .
$$


The function on the left-hand side and $w\left(x_{1}, x_{2}, x_{3}, x_{4}\right)$ defined by (4.4) can be obtained similarly to the single-observable case described in section 3.2. In fact it is a formidable task to search for solutions in the $4 \mathrm{~d}$ parameter space $\left(x_{1}, x_{2}, x_{3}, x_{4}\right)$ in full generality. However, given the insights we obtained from the analysis in the previous section, we can restrict ourselves to the region of $\left(x_{1}, x_{2}, x_{3}, x_{4}\right)$ in which we expect to find a solution. In particular, it is expected that there exist solutions which satisfy

$$
\begin{aligned}
& \text { (a) } x_{1}=x_{2}=x_{3}>1>x_{4}, \\
& \text { (b) } x_{1}=x_{2}>1>x_{3}>x_{4}, \\
& \text { (c) } x_{1}>1>x_{2}>x_{3}>x_{4} .
\end{aligned}
$$

The solutions one obtains for the cases (a) and (b) correspond to the $\mathrm{SO}(3)$ and $\mathrm{SO}(2)$ symmetric vacua, respectively, and hence one can compare the results against those obtained by the GEM with the $\mathrm{SO}(3)$ ansatz and the $\mathrm{SO}(2)$ ansatz. ${ }^{15}$ That would still require solving coupled equations for 2 variables in the case (a), and for 3 variables in the case (b). In what follows, we restrict ourselves to the $r=1$ case for simplicity, and investigate whether the GEM results for $\left\langle\tilde{\lambda}_{n}\right\rangle$ with the $\mathrm{SO}(3)$ ansatz and the $\mathrm{SO}(2)$ ansatz are indeed solutions to (4.9) in the cases (a) and (b), respectively. We also discuss the calculation of free energy in section 4.6 .

\section{2 analysis for the $\mathrm{SO}(3)$ symmetric vacuum}

Let us consider the case (a) in (4.10). We define the reduced functions

$$
\begin{gathered}
\rho_{\mathrm{SO}(3)}^{(0)}(x, y) \stackrel{\text { def }}{=} \rho^{(0)}(x, x, x, y), \\
w_{\mathrm{SO}(3)}(x, y) \stackrel{\text { def }}{=} w(x, x, x, y) .
\end{gathered}
$$

From the coupled equations (4.9), we obtain the counterpart of (3.17) as

$$
\frac{1}{N^{2}} f_{\mathrm{SO}(3), \zeta}^{(0)}(x, y)=-\frac{\partial}{\partial \zeta} \Phi_{\mathrm{SO}(3)}(x, y)
$$

where $\zeta=x, y$, and we have defined

$$
\begin{aligned}
& f_{\mathrm{SO}(3), \zeta}^{(0)}(x, y) \stackrel{\text { def }}{=} \frac{\partial}{\partial \zeta} \log \rho_{\mathrm{SO}(3)}^{(0)}(x, y), \\
& \Phi_{\mathrm{SO}(3)}(x, y) \stackrel{\text { def }}{=} \lim _{N \rightarrow \infty} \frac{1}{N^{2}} \log w_{\mathrm{SO}(3)}(x, y) .
\end{aligned}
$$

The GEM results obtained for $r=1$ with the $\mathrm{SO}(3)$ ansatz are $\left\langle\tilde{\lambda}_{n}\right\rangle_{\mathrm{SO}(3)}=1.17(n=1,2,3)$ and $\left\langle\tilde{\lambda}_{4}\right\rangle_{\mathrm{SO}(3)}=0.5$ (See Table 1.) We would like to check whether $(x, y)=(1.17,0.5)$ indeed solves eq. (4.13).

\footnotetext{
${ }^{15}$ The case (c) was not studied by GEM in ref. [35] since it is technically more difficult than the other cases due to the existence of more free parameters in the Gaussian action. However, our result $\Delta_{2}>0$ discussed in section 3.4 strongly suggests that the solution for the case (c) has larger free energy than the solution for the case (b).
} 
First let us consider the $\zeta=x$ component of eq. (4.13). Let us set $y$ to 0.5 and solve the equation for $x$ to see if the solution agrees with the value 1.17. We can calculate $w_{\mathrm{SO}(3)}(x, 0.5)$ and $f_{\mathrm{SO}(3), x}^{(0)}(x, 0.5)$ using the method described in section 3.2 . In fig. 4 (Left) we plot the function $\frac{1}{N^{2}} \log w_{\mathrm{SO}(3)}(x, 0.5)$ for $N=8,12,16$. We make a large- $N$ extrapolation using the asymptotic behavior at large $x$ to obtain $\Phi_{\mathrm{SO}(3)}(x, 0.5)$ represented by the solid lines. (See appendix D.1 for the details.) Figure 4 (Right) shows that the solution lies at $x=1.151(2)$, which is consistent with the GEM value 1.17.
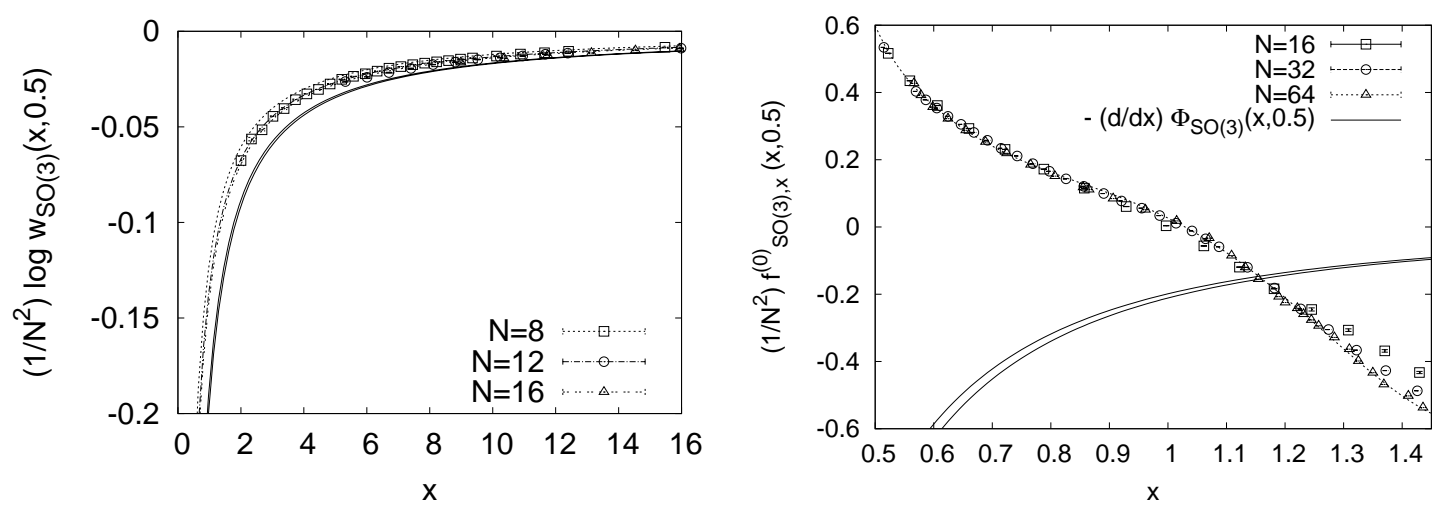

Figure 4: (Left) The function $\frac{1}{N^{2}} \log w_{\mathrm{SO}(3)}(x, 0.5)$ is plotted against $x$ for $N=8,12,16$. The solid lines represent the function $\Phi_{\mathrm{SO}(3)}(x, 0.5)$ obtained by extrapolation to $N=\infty$ as described in appendix D.1. (Right) The function $\frac{1}{N^{2}} f_{\mathrm{SO}(3), x}^{(0)}(x, 0.5)$ is plotted against $x$ for $N=16,32,64$. The solid lines represent $-\frac{\partial}{\partial x} \Phi_{\mathrm{SO}(3)}(x, 0.5)$ obtained from the plot on the left.

Next let us consider the $\zeta=y$ component of eq. (4.13). We set $x$ to 1.17 and solve the equation for $y$. In order to obtain $\Phi_{\mathrm{SO}(3)}(1.17, y)$, we have to make a large- $N$ extrapolation. For that purpose we first tried to use the asymptotic behavior of $\frac{1}{N^{2}} \log w_{\mathrm{SO}(3)}(1.17, y)$ at small $y$. However, it turned out that the finite- $N$ effects in $\frac{1}{N^{2}} \log w_{\mathrm{SO}(3)}(1.17, y)$ at small $y$ are much severer than in $\frac{1}{N^{2}} \log w_{\mathrm{SO}(3)}(x, 0.5)$ at large $x$ investigated above. ${ }^{16}$ We therefore use an "orthogonal" method here.

Note that we were able to obtain the function $\Phi_{\mathrm{SO}(3)}(x, y)$ at $y=0.5$ from fig. 4 (Left). We can do the same thing for different $y$ such as $y=0.45,0.55$. (See appendix D. 1 for the details.) In fig. 5 (Left) we plot $\Phi_{\mathrm{SO}(3)}(1.17, y)$ for $y=0.45,0.50,0.55$. By fitting the three points to a straight line, we obtain the derivative $\frac{\partial}{\partial y} \Phi_{\mathrm{SO}(3)}(1.17, y)=-0.33(2)$ at $y=0.5$. Figure 5 (Right) shows that the solution lies at $y=0.59(2)$, which is reasonably close to the GEM value 0.5 given the uncertainties involved in the analysis.

\footnotetext{
${ }^{16}$ The same trend can also be seen in the single-observable analysis relevant to the $\mathrm{SO}(3)$ ansatz. In fig. 2 (Left), the finite- $N$ effects in $\log w_{4}(x)$ at $x<1$ are much severer than in $\log w_{3}(x)$ at $x>1$.
} 

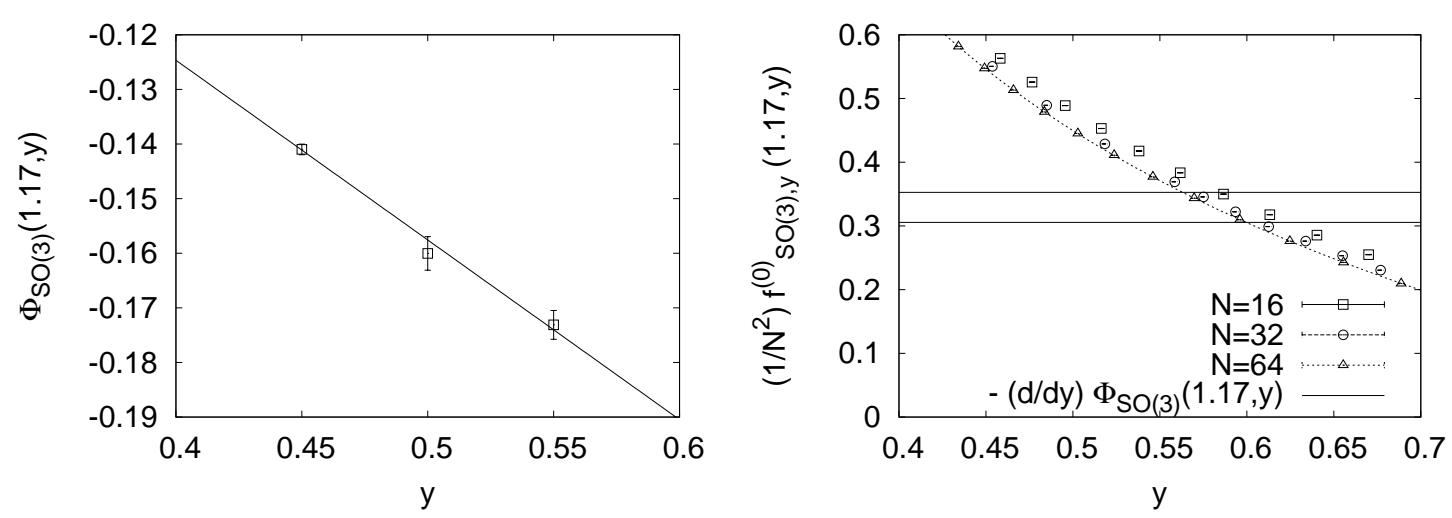

Figure 5: (Left) The values of the function $\Phi_{\mathrm{SO}(3)}(1.17, y)$ at $y=0.45,0.5,0.55$ are plotted against $y$. (Right) The function $\frac{1}{N^{2}} f_{\mathrm{SO}(3), y}^{(0)}(1.17, y)$ is plotted against $y$ for $N=16,32,64$. The solid lines represent $-\frac{\partial}{\partial y} \Phi_{\mathrm{SO}(3)}(1.17, y)$ at $y=0.5$ obtained from the plot on the left.

\section{3 analysis for the $\mathrm{SO}(2)$ symmetric vacuum}

Let us consider the case (b) in (4.10). We define the reduced functions

$$
\begin{gathered}
\rho_{\mathrm{SO}(2)}^{(0)}(x, y, z) \stackrel{\text { def }}{=} \rho^{(0)}(x, x, y, z), \\
w_{\mathrm{SO}(2)}(x, y, z) \stackrel{\text { def }}{=} w(x, x, y, z) .
\end{gathered}
$$

From the coupled equations (4.9), we obtain the counterpart of (3.17) as

$$
\frac{1}{N^{2}} f_{\mathrm{SO}(2), \zeta}^{(0)}(x, y, z)=-\frac{\partial}{\partial \zeta} \Phi_{\mathrm{SO}(2)}(x, y, z)
$$

where $\zeta=x, y, z$, and we have defined

$$
\begin{aligned}
f_{\mathrm{SO}(2), \zeta}^{(0)}(x, y, z) & \stackrel{\text { def }}{=} \frac{\partial}{\partial \zeta} \log \rho_{\mathrm{SO}(2)}^{(0)}(x, y, z) \\
\Phi_{\mathrm{SO}(2)}(x, y, z) & \stackrel{\text { def }}{=} \lim _{N \rightarrow \infty} \frac{1}{N^{2}} \log w_{\mathrm{SO}(2)}(x, y, z) .
\end{aligned}
$$

The GEM results obtained for $r=1$ with the $\mathrm{SO}(2)$ ansatz are $\left\langle\tilde{\lambda}_{n}\right\rangle_{\mathrm{SO}(2)}=1.4(n=$ $1,2),\left\langle\tilde{\lambda}_{3}\right\rangle_{\mathrm{SO}(2)}=0.7$ and $\left\langle\tilde{\lambda}_{4}\right\rangle_{\mathrm{SO}(2)}=0.5$ (See Table 1.) We would like to check whether $(x, y, z)=(1.4,0.7,0.5)$ indeed solves eq. (4.18).

First let us consider the $\zeta=x$ component of eq. (4.18). We set $y$ and $z$ to 0.7 and 0.5 , respectively, and solve the equation for $x$. In fig. 6 (Left) we plot the function $\frac{1}{N^{2}} \log w_{\mathrm{SO}(2)}(x, 0.7,0.5)$ for $N=8,12,16$. We make a large- $N$ extrapolation using the asymptotic behavior at large $x$ to obtain $\Phi_{\mathrm{SO}(2)}(x, 0.7,0.5)$ represented by the solid line. (See appendix D.2 for the details.) Figure 6 (Right) shows that the solution lies at $x=$ 1.373(2), which is consistent with the GEM value 1.4.

Next let us consider the $\zeta=y$ component of eq. (4.18). We set $x$ and $z$ to 1.4 and 0.5 , respectively, and solve the equation for $y$. In order to obtain $\Phi_{\mathrm{SO}(2)}(1.4, y, 0.5)$, we have to 

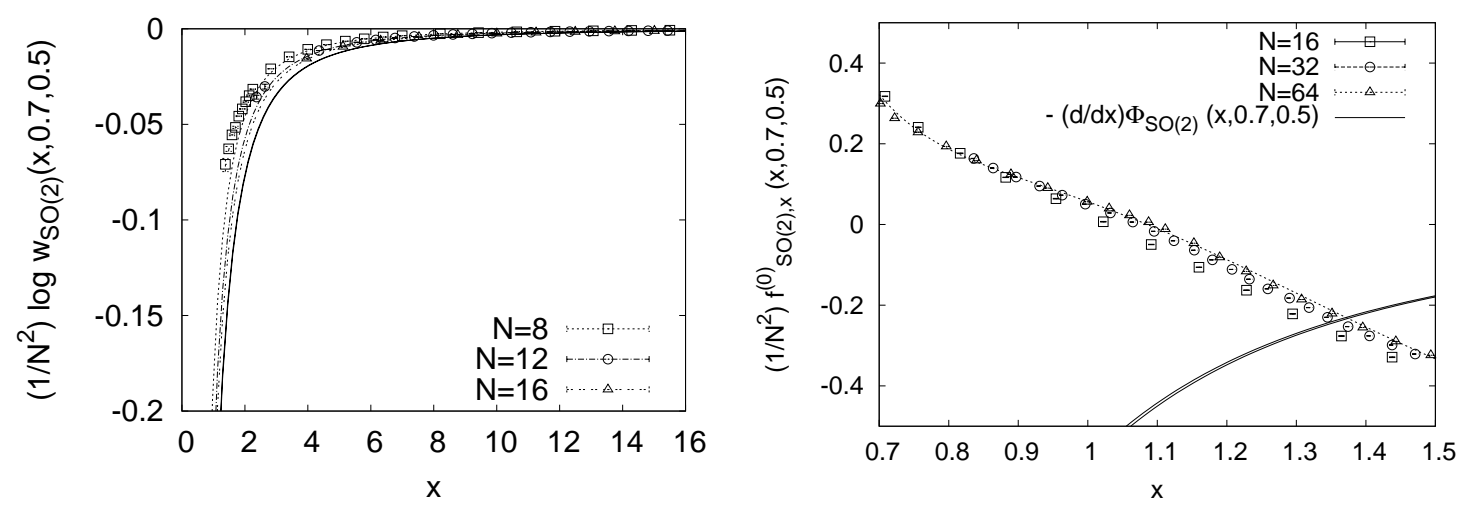

Figure 6: (Left) The function $\frac{1}{N^{2}} \log w_{\mathrm{SO}(2)}(x, 0.7,0.5)$ is plotted against $x$ for $N=8,12,16$. The solid line represents the function $\Phi_{\mathrm{SO}(2)}(x, 0.7,0.5)$ obtained by extrapolation to $N=\infty$ as described in appendix D.2. (Right) The function $\frac{1}{N^{2}} f_{\mathrm{SO}(2), x}^{(0)}(x, 0.7,0.5)$ is plotted against $x$ for $N=16,32,64$. The solid lines represent $-\frac{\partial}{\partial x} \Phi_{\mathrm{SO}(2)}(x, 0.7,0.5)$ obtained from the plot on the left.
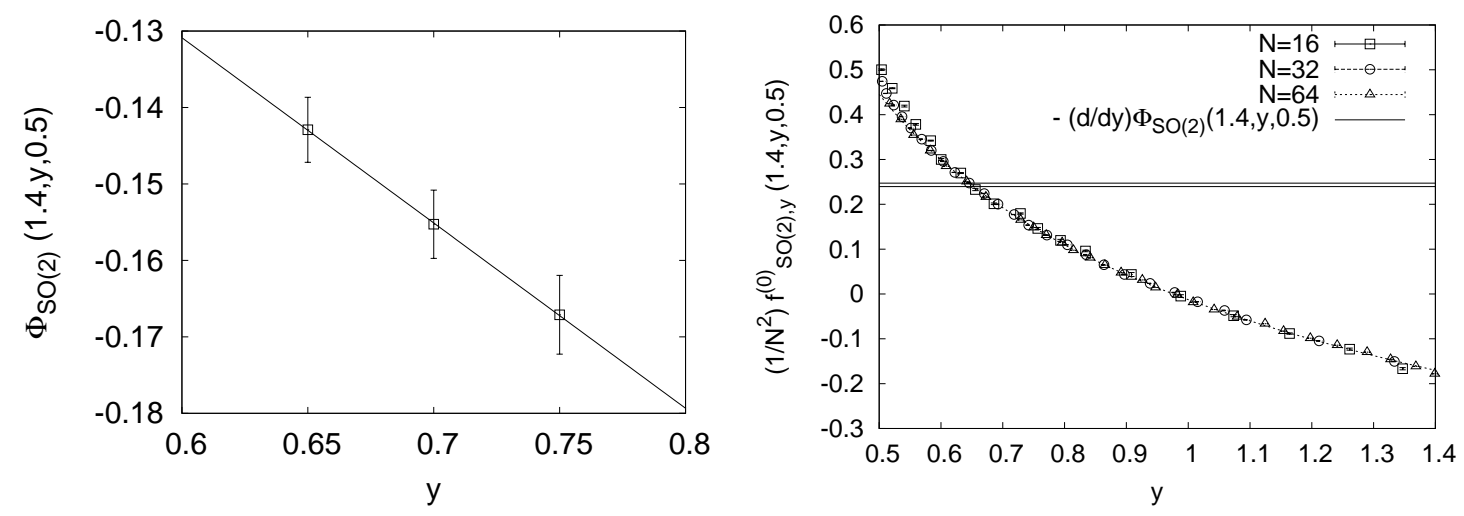

Figure 7: (Left) The values of the function $\Phi_{\mathrm{SO}(2)}(1.4, y, 0.5)$ at $y=0.65,0.70,0.75$ are plotted against $y$. (Right) The function $\frac{1}{N^{2}} f_{\mathrm{SO}(2), y}^{(0)}(1.4, y, 0.5)$ is plotted against $y$ for $N=16,32,64$. The solid lines represent $-\frac{\partial}{\partial y} \Phi_{\mathrm{SO}(2)}(1.4, y, 0.5)$ at $y=0.7$ obtained from the plot on the left.

make a large- $N$ extrapolation. The region of $y$ of the function $\frac{1}{N^{2}} \log w_{\mathrm{SO}(2)}(1.4, y, 0.5)$ is restricted to $0.5 \leq y \leq 1.4$, and we do not have any asymptotic behavior with respect to $y$ that can be used for large- $N$ extrapolation. Therefore we use the "orthogonal" method which was used in section 4.2 for studying the $\zeta=y$ component of eq. (4.13). Since we were able to obtain $\Phi_{\mathrm{SO}(2)}(x, y, z)$ for $y=0.7$ and $z=0.5$, we do the same thing for different $y$ such as $y=0.65,0.75$. In fig. 7 (Left) we plot $\Phi_{\mathrm{SO}(2)}(1.4, y, 0.5)$ for $y=0.65,0.70,0.75$. By fitting the three points to a straight line, we obtain the derivative $\frac{\partial}{\partial y} \Phi_{\mathrm{SO}(2)}(1.4, y, 0.5)=$ $-0.243(4)$ at $y=0.7$. Figure 7 (Right) shows that the solution lies at $y=0.649(4)$, which is consistent with the GEM value 0.7 given the uncertainties involved in the analysis.

Finally let us consider the $\zeta=z$ component of eq. (4.18). We set $x$ and $y$ to 1.4 and 

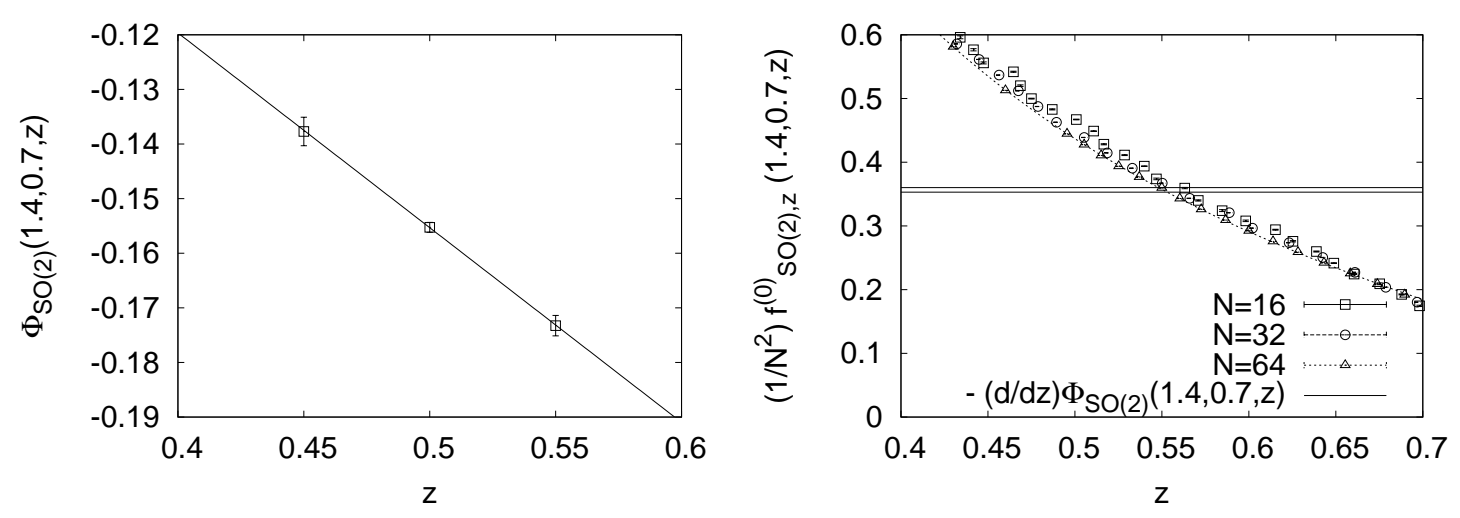

Figure 8: (Left) The values of the function $\Phi_{\mathrm{SO}(2)}(1.4,0.7, z)$ at $z=0.45,0.5,0.55$ are plotted against $z$. (Right) The function $\frac{1}{N^{2}} f_{\mathrm{SO}(2), z}^{(0)}(1.4,0.7, z)$ is plotted against $z$ for $N=16,32,64$. The solid lines represent $-\frac{\partial}{\partial z} \Phi_{\mathrm{SO}(2)}(1.4,0.7, z)$ at $z=0.5$ obtained from the plot on the left.

0.7, respectively, and solve the equation for $z$. In order to obtain $\Phi_{\mathrm{SO}(2)}(1.4,0.7, z)$, we use the "orthogonal" method. ${ }^{17}$ Since we were able to obtain $\Phi_{\mathrm{SO}(2)}(x, y, z)$ for $y=0.7$ and $z=0.5$, we do the same thing for different $z$ such as $z=0.45,0.55$. In fig. 8 (Left) we plot $\Phi_{\mathrm{SO}(2)}(1.4,0.7, z)$ at $z=0.45,0.50,0.55$. By fitting the three points to a straight line, we obtain the derivative $\frac{\partial}{\partial z} \Phi_{\mathrm{SO}(2)}(1.4,0.7, z)=-0.357(4)$ at $z=0.5$. Figure 8 (Right) shows that the solution lies at $z=0.551(2)$, which is close to the GEM value 0.5.

\section{4 estimates on the systematic error}

One thing we notice from the results in sections 4.2 and 4.3 (See Table 3 for a summary.) is that the agreement with the GEM results is better for the large eigenvalues $\left\langle\tilde{\lambda}_{n}\right\rangle>1$ than for the small eigenvalues $\left\langle\tilde{\lambda}_{n}\right\rangle<1$. Apart from the use of the "orthogonal" method in the latter case, we consider that the systematic error may be a possible reason.

Let us first consider the $\mathrm{SO}(3)$ symmetric vacuum. The $\mathrm{VEV}\left\langle\tilde{\lambda}_{n}\right\rangle$ is determined by solving (4.13). The left-hand side is determined accurately since there is no sign problem, and they behave as

$$
\begin{aligned}
& \frac{1}{N^{2}} f_{\mathrm{SO}(3), x}^{(0)}(x, 0.5) \sim-a(x-1.17)-b, \quad a=1.43(2), b=0.174(1) \\
& \frac{1}{N^{2}} f_{\mathrm{SO}(3), y}^{(0)}(1.17, y) \sim-\tilde{a}(y-0.5)+\tilde{b}, \quad \tilde{a}=1.70(4), \tilde{b}=0.452(1)
\end{aligned}
$$

near the solution as one can obtain from the right panel of figs. 4 and 5. Let us denote the right-hand side of the $\zeta$ component of (4.13) by $D_{\mathrm{SO}(3), \zeta}$, and assume that it has an error $\Delta D_{\mathrm{SO}(3), \zeta}$, where $\zeta=x, y$. This error includes the systematic error due to deviation from the asymptotic behavior as well as the one due to the large- $N$ extrapolation. Then

\footnotetext{
${ }^{17}$ We first tried to use the asymptotic behavior of $\frac{1}{N^{2}} \log w_{\mathrm{SO}(2)}(1.4,0.7, z)$ at small $z$. However, it turned out that the finite- $N$ effects in $\frac{1}{N^{2}} \log w_{\mathrm{SO}(2)}(1.4,0.7, z)$ at small $z$ are much severer than in $\frac{1}{N^{2}} \log w_{\mathrm{SO}(2)}(x, 0.7,0.5)$ at large $x$ investigated above.
} 
the error in $D_{\mathrm{SO}(3), x}$ propagates to the error of the solution $x$ as $a \Delta x=\Delta D_{\mathrm{SO}(3), x}$, from which we obtain

$$
\frac{\Delta x}{x}=\frac{\left|D_{\mathrm{SO}(3), x}\right|}{a x} \frac{\Delta D_{\mathrm{SO}(3), x}}{\left|D_{\mathrm{SO}(3), x}\right|} \sim 0.1 \frac{\Delta D_{\mathrm{SO}(3), x}}{\left|D_{\mathrm{SO}(3), x}\right|} .
$$

On the other hand, the error in $D_{\mathrm{SO}(3), y}$ propagates to the error of the solution $y$ as $\tilde{a} \Delta y=\Delta D_{\mathrm{SO}(3), y}$, from which we obtain

$$
\frac{\Delta y}{y}=\frac{\left|D_{\mathrm{SO}(3), y}\right|}{\tilde{a} y} \frac{\Delta D_{\mathrm{SO}(3), y}}{\left|D_{\mathrm{SO}(3), y}\right|} \sim 0.4 \frac{\Delta D_{\mathrm{SO}(3), y}}{\left|D_{\mathrm{SO}(3), y}\right|} .
$$

Thus we find that the coefficient is four times bigger for $y$ than for $x$. A similar analysis can be made for the $\mathrm{SO}(2)$ symmetric vacuum, and we obtain

$$
\begin{aligned}
& \frac{\Delta x}{x} \sim \frac{0.24}{0.82 \cdot 1.4} \frac{\Delta D_{\mathrm{SO}(2), x}}{\left|D_{\mathrm{SO}(2), x}\right|} \sim 0.21 \frac{\Delta D_{\mathrm{SO}(2), x}}{\left|D_{\mathrm{SO}(2), x}\right|}, \\
& \frac{\Delta y}{y} \sim \frac{0.25}{0.9 \cdot 0.7} \frac{\Delta D_{\mathrm{SO}(2), y}}{\left|D_{\mathrm{SO}(2), y}\right|} \sim 0.40 \frac{\Delta D_{\mathrm{SO}(2), y}}{\left|D_{\mathrm{SO}(2), y}\right|}, \\
& \frac{\Delta z}{z} \sim \frac{0.35}{1.6 \cdot 0.5} \frac{\Delta D_{\mathrm{SO}(2), z}}{\left|D_{\mathrm{SO}(2), z}\right|} \sim 0.43 \frac{\Delta D_{\mathrm{SO}(2), z}}{\left|D_{\mathrm{SO}(2), z}\right|},
\end{aligned}
$$

where $D_{\mathrm{SO}(2), \zeta}$ and $\Delta D_{\mathrm{SO}(2), \zeta}$ are defined analogously to the $\mathrm{SO}(3)$ case. Thus we find that the relative errors for the large eigenvalues tend to be smaller than those for the small eigenvalues.

\section{5 comparison with the single-observable analysis}

In this section we compare the results obtained above by the multi-observable analysis with those obtained by the single-observable analysis in section 3.4. For that, we first need to reconsider the interpretation of the latter taking into account the artifacts due to the remaining overlap problem.

Let us recall that the generalized distribution function $\rho\left(x_{1}, x_{2}, x_{3}, x_{4}\right)$ is expected to have three local maxima corresponding to each of the three cases (4.10). Therefore, the distribution function $\rho_{n}(x)$ in the single-observable analysis is also expected to have three local maxima due to the relation (4.6). The fact that we observe only two for $n=2,3$ and one for $n=1,4$ as we described in section 3.4 is due to the remaining overlap problem.

We reconsider the plots in the right column of fig. 2 from this point of view. For instance, the intersection of the two curves shown in the uppermost panel can be naturally identified as the one corresponding to the case (c). As $x$ decreases from the intersecting point, the true curve $y=-\frac{d}{d x} \Phi_{1}(x)$ is expected to have four more intersections with the curve $y=\frac{1}{N^{2}} f_{1}^{(0)}(x)$ in the $x>1$ region. Two of them represent the local maxima of the distribution function $\rho_{1}(x)$ corresponding to the cases (a) and (b) in (4.10). Such a structure is invisible in the results of the single-observable analysis due to the overlap problem. From this point of view, it is misleading to compare the solution $x_{1}$ for the $n=1$ case with the $\left\langle\tilde{\lambda}_{1}\right\rangle$ for the $\mathrm{SO}(2)$ symmetric vacuum. Rather we should consider it as an estimate for $\left\langle\tilde{\lambda}_{1}\right\rangle$ in the vacuum corresponding to the case (c) in (4.10). This provides a prediction for the calculation using GEM, which is not done yet. (See footnote 15.) 
Similar arguments apply to the $n=2,3,4$ cases. The solutions $x_{\mathrm{s}}$ and $x_{1}$ for the $n=2$ case should be interpreted as estimates on $\left\langle\tilde{\lambda}_{2}\right\rangle$ in the vacuum corresponding to the case (c) in (4.10) and in the $\mathrm{SO}(2)$ symmetric vacuum, respectively. The solutions $x_{\mathrm{S}}$ and $x_{1}$ for the $n=3$ case should be interpreted as estimates on $\left\langle\tilde{\lambda}_{3}\right\rangle$ for the $\mathrm{SO}(2)$ and $\mathrm{SO}(3)$ symmetric vacua, respectively. The solution $x_{\mathrm{s}}$ for the $n=4$ case should be interpreted as an estimate on $\left\langle\tilde{\lambda}_{4}\right\rangle$ for the $\mathrm{SO}(3)$ symmetric vacuum.

In Table 3 we summarize our results for $\left\langle\tilde{\lambda}_{n}\right\rangle$ corresponding to the $\mathrm{SO}(2)$ and $\mathrm{SO}(3)$ symmetric vacua. We also show the results of the single-observable analysis with the new interpretation except for an estimate on $\left\langle\tilde{\lambda}_{4}\right\rangle$ for the $\mathrm{SO}(2)$ symmetric vacuum, which is not available. We do find that the results of the multi-observable analysis have better agreement with the GEM values.

\begin{tabular}{|c|c|c|c|c|c|c|}
\hline ansatz & \multicolumn{3}{|c|}{$\mathrm{SO}(3)$} & \multicolumn{3}{c|}{$\mathrm{SO}(2)$} \\
\hline method & single-obs. & multi-obs. & GEM & single-obs. & multi-obs. & GEM \\
\hline$\left\langle\tilde{\lambda}_{1}\right\rangle$ & - & - & 1.17 & - & - & 1.4 \\
$\left\langle\tilde{\lambda}_{2}\right\rangle$ & - & - & 1.17 & $1.317(1)$ & $1.373(2)$ & 1.4 \\
$\left\langle\tilde{\lambda}_{3}\right\rangle$ & $1.129(1)$ & $1.151(2)$ & 1.17 & $0.62(2)$ & $0.649(4)$ & 0.7 \\
$\left\langle\tilde{\lambda}_{4}\right\rangle$ & $0.71(5)$ & $0.59(2)$ & 0.5 & not available & $0.551(2)$ & 0.5 \\
\hline
\end{tabular}

Table 3: The results for the normalized eigenvalues $\left\langle\tilde{\lambda}_{n}\right\rangle$ for $r=1$ obtained by the factorization method with the single-observable and multi-observable analyses for the $\mathrm{SO}(3)$ and $\mathrm{SO}(2)$ symmetric vacua. The dash implies that the result should be the same as the one below in the same column due to the imposed symmetry. We also show the GEM results obtained at $N=\infty$ in ref. [35].

\section{6 calculation of the free energy}

In sections 4.2 and 4.3, we have checked that the GEM results with the $\mathrm{SO}(3)$ ansatz and the $\mathrm{SO}(2)$ ansatz can indeed be obtained as solutions to (4.9). In order to determine which solution dominates the path integral, we need to compare the free energy. This was done in the GEM and the result is shown in Table 1, from which we find that the $\mathrm{SO}(2)$ symmetric solution dominates in the large- $N$ limit. Here we try to obtain the difference of free energy for the two solutions by Monte Carlo simulation. The factorization method has the nice feature that the difference of free energy is decomposed into the term coming from the phase-quenched model and the term due to the effect of the phase. The former can be calculated accurately, and the latter is the main source of the error.

What we should calculate is the difference of $\log \rho\left(x_{1}, x_{2}, x_{3}, x_{4}\right)$ between the two solutions. Let us denote the solution for cases (a) and (b) as $\vec{x}_{a}=\left(X^{\prime}, X^{\prime}, X^{\prime}, Y^{\prime}\right)$ and $\vec{x}_{b}=(X, X, Y, Z)$, respectively. From Table 1 we find for $r=1$ that

$$
\begin{aligned}
& \text { (a) } X^{\prime} \simeq 1.17, Y^{\prime} \simeq 0.5 \\
& \text { (b) } X \simeq 1.4, Y \simeq 0.7, Z \simeq 0.5 .
\end{aligned}
$$


Using the factorization property (4.3), we obtain

$$
\begin{aligned}
& \Delta \stackrel{\text { def }}{=} \frac{1}{N^{2}}\left\{\log \rho\left(\vec{x}_{a}\right)-\log \rho\left(\vec{x}_{b}\right)\right\} \\
&=\left\{\Phi_{\mathrm{SO}(3)}\left(X^{\prime}, Y^{\prime}\right)-\Phi_{\mathrm{SO}(2)}(X, Y, Z)\right\}+\Xi, \\
& \text { where } \quad \Xi \stackrel{\text { def }}{=} \int_{\vec{x}_{b}}^{\vec{x}_{a}} d x_{j} \frac{1}{N^{2}} \frac{\partial}{\partial x_{j}} \log \rho^{(0)}\left(x_{1}, x_{2}, x_{3}, x_{4}\right) .
\end{aligned}
$$

The first term in (4.30) can be estimated as (See appendix D.)

$$
\begin{aligned}
\Phi_{\mathrm{SO}(3)}(1.17,0.5) & =-0.160(3) \\
\Phi_{\mathrm{SO}(2)}(1.4,0.7,0.5) & =-0.155(1) .
\end{aligned}
$$

In order to calculate the second term $\Xi$ in (4.30), we first define a path in the $\left(x_{1}, x_{2}, x_{3}, x_{4}\right)$ space connecting the two solutions $\vec{x}_{a}$ and $\vec{x}_{b}$, obtain the gradient of $\log \rho^{(0)}\left(x_{1}, x_{2}, x_{3}, x_{4}\right)$ and integrate it along the path. As a path connecting the two solutions, we consider

$$
\begin{aligned}
& C_{1}:(X, X, Y, Z) \mapsto(X, X, X, Z), \\
& C_{2}:(X, X, X, Z) \mapsto\left(X, X, X, Y^{\prime}\right), \\
& C_{3}:\left(X, X, X, Y^{\prime}\right) \mapsto\left(X^{\prime}, X^{\prime}, X^{\prime}, Y^{\prime}\right) .
\end{aligned}
$$

The paths $C_{1}$ and $C_{3}$ are investigated already in sections 4.3 and 4.2 , respectively, while the path $C_{2}$ should be investigated newly. In the $r=1$ case, we have $Y \simeq Z^{\prime}$ accidentally, and therefore the study of the path $C_{2}$ can be totally omitted. The second term in (4.30) can then be evaluated as

$$
\begin{aligned}
\Xi & =\int_{0.7}^{1.4} \frac{1}{N^{2}} f_{\mathrm{SO}(2), y}^{(0)}(1.4, y, 0.5) d y-\int_{1.17}^{1.4} \frac{1}{N^{2}} f_{\mathrm{SO}(3), x}^{(0)}(x, 0.5) d x \\
& =(-0.014)-(-0.079)=0.065,
\end{aligned}
$$

where the integrals are calculated by fitting the data points for largest $N$ in figs. 4 and 7 (Right) to some known functions with free parameters in the integration domain. The errors are negligible compared to those in the first term of (4.30). Thus we obtain $\Delta=0.060(4)$, which should be compared with the value $\Delta \simeq-0.3$ predicted by GEM as one can see from Table 1.

According to the interpretation of the single-observable analysis given in section 4.5, what we have done above may be regarded as a refined version of the calculation of $\Delta_{3}$ in section 3.4, where we obtained $\Delta_{3}=0.11(4)$ for $r=1$.

Let us recall that the calculation of (4.34) does not have much ambiguity. Note also that $\Xi>0$ implies that the $\mathrm{SO}(3)$ symmetric configuration $\left(X^{\prime}, X^{\prime}, X^{\prime}, Y^{\prime}\right)$ has lower free energy in the phase-quenched model than the $\mathrm{SO}(2)$ symmetric configuration $(X, X, Y, Z)$. This is reasonable considering that the former configuration is closer to the dominant $\mathrm{SO}(4)$ symmetric configuration $\tilde{\lambda}_{n}=1$ in the phase-quenched model.

On the other hand, the estimates (4.32) and (4.33) may be subject to systematic errors due to the deviation of the functions $\Phi_{\mathrm{SO}(3)}(x, 0.5)$ and $\Phi_{\mathrm{SO}(2)}(x, 0.7,0.5)$ from their 
asymptotic behaviors and due also to large- $N$ extrapolations. Let us recall that the error propagation in solving the $\zeta=x$ component of eq. (4.13) is given by (4.23), while the error in (4.32) can be roughly estimated to be

$$
\frac{\Delta \Phi_{\mathrm{SO}(3)}(1.17,0.5)}{\left|\Phi_{\mathrm{SO}(3)}(1.17,0.5)\right|} \sim \frac{\Delta D_{\mathrm{SO}(3), x}}{\left|D_{\mathrm{SO}(3), x}\right|}
$$

Therefore, the relative error in $\Phi_{\mathrm{SO}(3)}(1.17,0.5)$ is ten times larger than that in $\left\langle\tilde{\lambda}_{3}\right\rangle$ in the $\mathrm{SO}(3)$ symmetric vacuum. The crucial point here is that the function $\Phi_{\mathrm{SO}(3)}(x, 0.5)$ changes very rapidly in the region where the solution to (4.13) lies. Therefore, a small systematic error in its estimation affects the value at the solution much more than the solution itself. A similar argument applies also to the $\mathrm{SO}(2)$ symmetric vacuum. It is therefore reasonable to conclude that the first term in (4.30) is not obtained with the accuracy that is sufficient for the determination of the sign of $\Delta$.

While this sounds a bit disappointing, let us recall that the $\mathrm{SO}(2)$ and $\mathrm{SO}(3)$ symmetric vacua become degenerate as the parameter $r \equiv N_{\mathrm{f}} / N$ of the model goes to zero. For instance, it would have been much easier to compare the free energy for the solutions corresponding to the cases (b) and (c) in (4.10) as the calculation of $\Delta_{2}$ in section 3.4 suggests. Clearly the situation would be model dependent, and we consider that the difficulty in the calculation of the free energy is not a generic feature of the factorization method.

\section{7 including more observables}

As we have seen above, the overlap problem can be reduced drastically by constraining all the $\tilde{\lambda}_{n}(n=1,2,3,4)$ at the same time instead of constraining just one of them. A natural question that arises then is whether there is no more overlap problem we have to worry about so that we actually do not need to include more observables in our analysis. Note that this is relevant not only to the calculation of the VEV of observables other than $\tilde{\lambda}_{n}$, but also to the calculation of $\left\langle\tilde{\lambda}_{n}\right\rangle$ and the free energy that we have discussed above.

Let us see directly a possible overlap problem associated with a general observable $\mathcal{O}$ in a simulation with constraints on $\tilde{\lambda}_{n}(n=1,2,3,4)$. For that we rewrite the $\operatorname{VEV}\langle\mathcal{O}\rangle$ as

$$
\begin{aligned}
\langle\mathcal{O}\rangle & =\int \prod_{k=1}^{4} d x_{k}\left\langle\mathcal{O} \prod_{k=1}^{4} \delta\left(x_{k}-\tilde{\lambda}_{k}\right)\right\rangle \\
& =\int \prod_{k=1}^{4} d x_{k} \rho\left(x_{1}, x_{2}, x_{3}, x_{4}\right) \frac{\left\langle\mathcal{O} \mathrm{e}^{i \Gamma}\right\rangle_{x_{1}, x_{2}, x_{3}, x_{4}}}{\left\langle\mathrm{e}^{i \Gamma}\right\rangle_{x_{1}, x_{2}, x_{3}, x_{4}}}
\end{aligned}
$$

Assuming the usual equivalence between the canonical ensemble and the microcanonical ensemble, we may consider that the integral over $x_{k}$ in (4.37) is dominated by $(X, X, Y, Z)$ giving the absolute maximum of $\rho\left(x_{1}, x_{2}, x_{3}, x_{4}\right)$. This leads to

$$
\langle\mathcal{O}\rangle \simeq \frac{\left\langle\mathcal{O} \mathrm{e}^{i \Gamma}\right\rangle_{X, X, Y, Z}}{\left\langle\mathrm{e}^{i \Gamma}\right\rangle_{X, X, Y, Z}}
$$


Thus we can calculate the VEV by using the standard reweighting formula within the "microcanonical ensemble" characterized by $(X, X, Y, Z)$. For $\mathcal{O}=\tilde{\lambda}_{n}$, the VEV will be given by $X, X, Y, Z$ for $n=1,2,3,4$, respectively, as it should.

For a general operator $\mathcal{O}$, however, we can still have an overlap problem due to the same reason that we have one when we apply the reweighting method to the original model. If it turns out that $\langle\mathcal{O}\rangle_{X, X, Y, Z}$ is actually close to (4.38), there is no more overlap problem as far as the operator $\mathcal{O}$ is concerned. Note that this requires that

$$
\left\langle\mathcal{O} \mathrm{e}^{i \Gamma}\right\rangle_{X, X, Y, Z} \sim\langle\mathcal{O}\rangle_{X, X, Y, Z}\left\langle\mathrm{e}^{i \Gamma}\right\rangle_{X, X, Y, Z}
$$

as we can see from (4.38). Eq. (4.39) only implies that the correlation of the operator $\mathcal{O}$ and the phase factor $\mathrm{e}^{i \Gamma}$ within the "microcanonical ensemble" is small. If the same statement holds for arbitrary observable, we may say that the sign problem is practically solved even if $\left\langle\mathrm{e}^{i \Gamma}\right\rangle_{X, X, Y, Z}$ is not close to one at all.

Thus we find that the success of the factorization method in a general model relies on whether one can find the minimal set of observables that is needed to "solve" the sign problem in the above sense [36]. We consider that the matrix model we are studying provides an explicit example in which this can be done.

In order to provide some evidence for this statement, let us consider an observable

$$
\mathcal{O}=-\frac{1}{N} \sum_{\mu \neq \nu} \operatorname{tr}\left[A_{\mu}, A_{\nu}\right]^{2}
$$

and the normalized one $\widetilde{\mathcal{O}}=\mathcal{O} /\langle\mathcal{O}\rangle_{0}$. When we constrain $\widetilde{\mathcal{O}}$ to a small value, the dominant configurations have the property $\left[A_{\mu}, A_{\nu}\right] \approx 0$, meaning that $A_{\mu}$ are simultaneously diagonalizable, e.g. as $A_{\mu}=\operatorname{diag}\left(\alpha_{\mu}^{(1)}, \cdots, \alpha_{\mu}^{(N)}\right)$. For such configurations, the determinant becomes

$$
\operatorname{det} \mathcal{D}=\prod_{i=1}^{N}\left\{\sum_{\mu}\left(\alpha_{\mu}^{(i)}\right)^{2}\right\} \geq 0 \text {. }
$$

Therefore, the observable (4.40) is considered as a "dangerous" one, which can potentially have strong correlation with the phase factor.

Clearly the formula (4.38) is not useful for actually investigating the overlap problem associated with the operator $\mathcal{O}$. For that purpose, we can use the factorization method including not only $\tilde{\lambda}_{n}(n=1,2,3,4)$ but also $\widetilde{\mathcal{O}}$. Let us define the corresponding functions $\rho^{(0)}$ and $w$ with five arguments, and also define the reduced functions

$$
\begin{aligned}
\rho_{\mathcal{O}}^{(0)}(x) & =\rho^{(0)}(X, X, Y, Z, x), \\
w_{\mathcal{O}}(x) & =w(X, X, Y, Z, x) .
\end{aligned}
$$

The VEV $\langle\widetilde{\mathcal{O}}\rangle$ can then be obtained by solving

$$
\frac{1}{N^{2}} f_{\mathcal{O}}^{(0)}(x)=-\frac{d}{d x} \Phi_{\mathcal{O}}(x)
$$



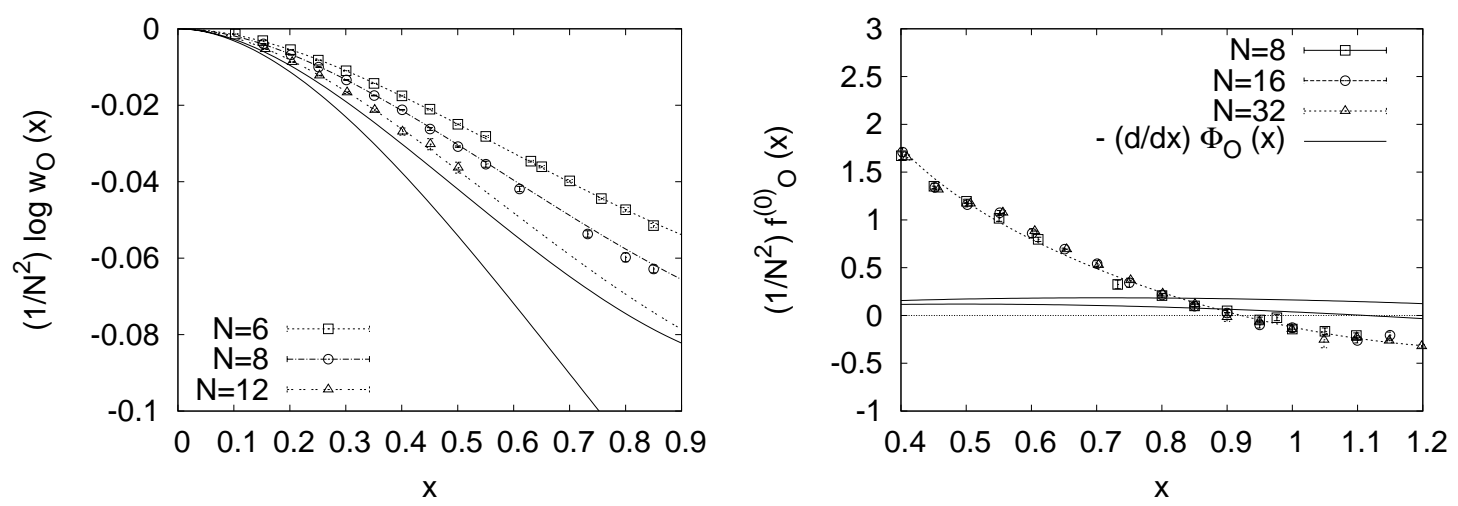

Figure 9: (Left) The function $\frac{1}{N^{2}} \log w_{\mathcal{O}}(x)$ is plotted against $x$ for $N=6,8,12$. We also plot the function $\Phi_{\mathcal{O}}(x)$ obtained by extrapolation to $N=\infty$ as described in appendix D.2. The two solid lines represent the margin of error. (Right) The function $\frac{1}{N^{2}} \frac{d}{d x} \log \rho_{\mathcal{O}}^{(0)}(x)$ is plotted for $N=8,16,32$. We also plot $-\frac{d}{d x} \Phi_{\mathcal{O}}(x)$ obtained from the plot on the left.

where $f_{\mathcal{O}}^{(0)}(x)$ and $\Phi_{\mathcal{O}}(x)$ are defined as

$$
\begin{aligned}
f_{\mathcal{O}}^{(0)}(x) & =\frac{d}{d x} \log \rho_{\mathcal{O}}^{(0)}(x) \\
\Phi_{\mathcal{O}}(x) & =\lim _{N \rightarrow \infty} \frac{1}{N^{2}} \log w_{\mathcal{O}}(x) .
\end{aligned}
$$

From fig. 9 (Left) we find that $\frac{1}{N^{2}} \log w_{\mathcal{O}}(x)$ approaches zero for $x \rightarrow 0$ as expected from (4.41). We make a large- $N$ extrapolation as described in appendix D.2 to obtain $\Phi_{\mathcal{O}}(x)$, which is also shown in the same figure by the two solid lines showing the margin of error. From fig. 9 (Right) we find that the effect of the phase is to shift the estimate of $\langle\widetilde{\mathcal{O}}\rangle$ by $\Delta x=0.07(3)$. On the other hand, the standard deviation of the distribution $\rho_{\mathcal{O}}^{(0)}(x)$ is estimated as $\sigma \sim 0.7 / N$ from the slope of the function plotted in fig. 9 (Right) around $x \sim 0.92$. This means that the deviation $\Delta x$ is $\lesssim 2 \sigma$ for $N \leq 16$. Thus, the remaining overlap problem associated with this observable (4.40) is practically small. This is consistent with the fact that we were able to reproduce the GEM result by constraining only the four observables $\lambda_{n}(n=1,2,3,4)$.

\section{Summary and discussions}

In this paper we tested the proposed scenario for dynamical compactification of spacetime in the IIB matrix model, in which the phase of the fermion determinant induces the spontaneous breaking of rotational symmetry. We have shown that this can indeed occur in a simplified model [34] by performing Monte Carlo simulations. The model exhibits SSB of $\mathrm{SO}(4)$ rotational symmetry in agreement with the results of GEM [35], and the phase of the fermion determinant plays a crucial role in the mechanism of SSB.

First, in the absence of the phase, we have confirmed that the model has no SSB by calculating the $\mathrm{VEV}$ of the eigenvalues of $T_{\mu \nu}$, which is analogous to the moment of 
inertia tensor. The effect of the phase has been studied by using the factorization method originally proposed in ref. [24]. From the single-observable analysis, we find that the phase fluctuations strongly suppress the region of the configuration space favored in the phase quenched model and result in different length scales for each dimension of space-time. While our results are in partial agreement with the GEM results in ref. [35], we also observe some discrepancies and puzzles, which we attributed to the remaining overlap problem. This motivated us to generalize the method to multiple observables. In particular, we find that controlling the four eigenvalues of the "moment of inertia tensor" is good enough to reduce the overlap problem to a sufficient level. Restricting ourselves to the $\mathrm{SO}(2)$ and $\mathrm{SO}(3)$ symmetric vacua, we were able to reproduce the GEM results for the VEV of the eigenvalues consistently.

Our results are an encouragement for pursuing similar studies on the IIB matrix model. Although it is computationally more demanding, the supersymmetry of the IIB matrix model could make the SSB of rotational symmetry easier to see. In particular, we have observed in ref. [24] that the $\frac{1}{N^{2}} f_{n}^{(0)}(x)$ that appears in (3.17) is actually very close to zero, which can be understood as a result of cancellations by fermionic and bosonic contributions to the interactions among space-time points. This implies that the solutions to eq. (3.17) appear in the region where the sign problem is not severe. We are currently studying the 6 dimensional version of the IIB matrix model by Monte Carlo simulation [37] and trying to compare the results against the predictions obtained by the GEM recently [38].

We would like to emphasize that the model studied in this paper has a severe sign problem despite its simpleness. It is encouraging that such a system can be studied by Monte Carlo simulation using the factorization method, which gives us a lot of useful insights into the effect of the phase. The method itself is quite general and it can be applied to any interesting system which suffers from the sign problem. The crucial step is to find an appropriate set of observables that one can control in order to determine and sample efficiently the region of the configuration space favored by the relatively small fluctuation of the phase. We expect that this is possible in many interesting systems.

\section{Acknowledgments}

The authors would like to thank Shinji Ejiri, Sourendu Gupta and Yuko Okamoto for valuable discussions. This work was partially funded by the National Technical University of Athens through the "Basic Research Support Programme 2009 and 2010". The work of T.A. and J.N. was supported in part by Grant-in-Aid for Scientific Research (No. 03740, 23740211 for T.A. and 20540286 for J.N.) from Japan Society for the Promotion of Science.

\section{A. Details of the Monte Carlo simulation}

In this section we present the details of the algorithm used for our Monte Carlo simulation. It is essentially the hybrid Monte Carlo (HMC) algorithm, which has been applied in similar models in refs. [21, 22, 24, 25,39]. The computational effort grows as $\mathrm{O}\left(N^{3}\right)$ in the present model. 
We first discuss the HMC algorithm for the phase-quenched model (2.12). We introduce auxiliary bosonic Hermitian matrices $P_{\mu}$ and consider the action

$$
S_{\mathrm{HMC}}[P, A]=\frac{1}{2} \operatorname{tr}\left(P_{\mu}\right)^{2}+S_{0}[A] .
$$

The original model is obtained by integrating out $P_{\mu}$. We regard the action $S_{\mathrm{HMC}}[P, A]$ as the Hamiltonian of a classical system described by $A_{\mu}(\tau)$ and its conjugate momentum $P_{\mu}(\tau)$, where $\tau$ denotes the fictitious time of the classical system. The Hamiltonian equation of motion is obtained as

$$
\begin{aligned}
\frac{d\left(A_{\mu}\right)_{k l}}{d \tau} & =\frac{\partial S_{\mathrm{HMC}}}{\partial\left(P_{\mu}\right)_{k l}}=\left(P_{\mu}\right)_{l k}, \\
\frac{d\left(P_{\mu}\right)_{k l}}{d \tau} & =-\frac{\partial S_{\mathrm{HMC}}}{\partial\left(A_{\mu}\right)_{k l}} \\
& =-N\left(A_{\mu}\right)_{l k}+\frac{N_{f}}{2}\left\{\operatorname{Tr}\left(\mathcal{D}^{-1} \frac{\partial \mathcal{D}}{\partial\left(A_{\mu}\right)_{k l}}\right)+\operatorname{Tr}\left(\mathcal{D}^{-1} \frac{\partial \mathcal{D}}{\partial\left(A_{\mu}\right)_{l k}}\right)^{*}\right\},
\end{aligned}
$$

where $\operatorname{Tr}$ denotes a trace with respect to the $2 N$-dimensional index. The indices $k, l$ run over $k, l=1,2, \cdots, N$. The updating procedure consists of (i) refreshing the momentum variables $P_{\mu}$ by Gaussian random numbers, which obey the distribution $e^{-S_{\mathrm{HMC}}}$ and (ii) solving the Hamiltonian equation of motion for a fixed time interval $\tau$. In actual calculations we have to discretize the Hamiltonian equation (A.2) and (A.3). The reversibility of the time evolution is preserved by using the so-called leap-frog discretization, which gives

$$
\begin{aligned}
\left(P_{\mu}^{(1 / 2)}\right)_{k l} & =\left(P_{\mu}^{(0)}\right)_{k l}-\frac{\Delta \tau}{2} \frac{\partial S_{\mathrm{HMC}}}{\partial\left(A_{\mu}\right)_{k l}}\left(A_{\mu}^{(0)}\right), \\
\left(A_{\mu}^{(1)}\right)_{k l} & =\left(A_{\mu}^{(0)}\right)_{k l}+\Delta \tau\left(P_{\mu}^{(1 / 2)}\right)_{l k}, \\
\left(P_{\mu}^{(r+1 / 2)}\right)_{k l} & =\left(P_{\mu}^{(r-1 / 2)}\right)_{k l}-\Delta \tau \frac{\partial S_{\mathrm{HMC}}}{\partial\left(A_{\mu}\right)_{k l}}\left(A_{\mu}^{(r)}\right), \\
\left(A_{\mu}^{(r+1)}\right)_{k l} & =\left(A_{\mu}^{(r)}\right)_{k l}+\Delta \tau\left(P_{\mu}^{(r+1 / 2)}\right)_{l k}, \\
\left(P_{\mu}^{(\nu)}\right)_{k l} & =\left(P_{\mu}^{(\nu-1 / 2)}\right)_{k l}-\frac{\Delta \tau}{2} \frac{\partial S_{\mathrm{HMC}}}{\partial\left(A_{\mu}\right)_{k l}}\left(A_{\mu}^{(\nu)}\right),
\end{aligned}
$$

where $r=1,2, \cdots, \nu-1$ and $T=\nu \Delta \tau$, and we have introduced the short-hand notation $P_{\mu}^{(r)}=P_{\mu}(r \Delta \tau)$ and $A_{\mu}^{(r)}=A_{\mu}(r \Delta \tau)$. The conservation of the Hamiltonian $\left(S_{\mathrm{HMC}}\right)$ is violated by the discretization. The detailed balance can be preserved, however, by adding a Metropolis accept/reject procedure at the end of each trajectory. Namely we accept the trial configuration with the probability $\min \left(1, e^{-\Delta S_{\mathrm{HMC}}}\right)$, where $\Delta S_{\mathrm{HMC}}$ represents the difference of $S_{\mathrm{HMC}}$ between the trial and original configurations. The step size $\Delta \tau$ for the time evolution should be small enough to keep the acceptance rate reasonably high.

Next we discuss how to simulate the constrained system defined by the partition function $Z_{x_{1}, x_{2}, x_{3}, x_{4}}$ in eq. (4.5). As we discussed in section 3.2 for the single-observable analysis, 
we approximate the delta function by the Gaussian function as

$$
\begin{aligned}
Z_{x_{1}, x_{2}, x_{3}, x_{4}, V} & =\int d A \exp \left[-\left(S_{0}+\sum_{n=1}^{4} V_{n}\left(\lambda_{n}\right)\right)\right] \\
V_{n}\left(\lambda_{n}\right) & =\frac{1}{2} \gamma_{n}\left(\lambda_{n}-\xi_{n}\right)^{2}
\end{aligned}
$$

The system (3.10) used for the single-observable analysis in section 3 can be obtained by setting $\gamma_{k}=0$ for $k \neq n$.

Taking into account the potential term $V_{n}\left(\lambda_{n}\right)$ in (A.7), we need to subtract the term $\frac{\partial V_{n}\left(\lambda_{n}\right)}{\partial\left(A_{\mu}\right)_{k l}}$ from the right-hand side of eq. (A.3). This term can be calculated explicitly as follows. Let us note first that the eigenvalues $\lambda_{n}$ satisfy

$$
\sum_{\rho=1}^{4} T_{\nu \rho} v_{\rho}^{(n)}=\lambda_{n} v_{\nu}^{(n)}
$$

where $v_{\mu}^{(n)}$ is the eigenvector of the $4 \times 4$ matrix $T_{\mu \nu}$ normalized as $\sum_{\mu=1}^{4} v_{\mu}^{(n)} v_{\mu}^{(n)}=1$. (No summation over the index $n$.) Taking the derivative of eq. (A.9) with respect to $\left(A_{\mu}\right)_{k l}$, we obtain

$$
\sum_{\rho=1}^{4}\left(\frac{\partial T_{\nu \rho}}{\partial\left(A_{\mu}\right)_{k l}} v_{\rho}^{(n)}+T_{\nu \rho} \frac{\partial v_{\rho}^{(n)}}{\partial\left(A_{\mu}\right)_{k l}}\right)=\frac{\partial \lambda_{n}}{\partial\left(A_{\mu}\right)_{k l}} v_{\nu}^{(n)}+\lambda_{n} \frac{\partial v_{\nu}^{(n)}}{\partial\left(A_{\mu}\right)_{k l}} .
$$

Multiplying both sides of eq. (A.10) by $v_{\nu}^{(n)}$ and taking a sum over $\nu$, we obtain

$$
\sum_{\nu, \rho=1}^{4} v_{\nu}^{(n)} \frac{\partial T_{\nu \rho}}{\partial\left(A_{\mu}\right)_{k l}} v_{\rho}^{(n)}=\sum_{\nu=1}^{4} v_{\nu}^{(n)} \frac{\partial \lambda_{n}}{\partial\left(A_{\mu}\right)_{k l}} v_{\nu}^{(n)}=\frac{\partial \lambda_{n}}{\partial\left(A_{\mu}\right)_{k l}},
$$

where the second terms of each side of eq. (A.10) cancel. Therefore we obtain

$$
\frac{\partial V_{n}\left(\lambda_{n}\right)}{\partial\left(A_{\mu}\right)_{k l}}=\gamma_{n}\left(\lambda_{n}-\xi_{n}\right) \frac{\partial \lambda_{n}}{\partial\left(A_{\mu}\right)_{k l}}=\frac{2 \gamma_{n}}{N}\left(\lambda_{n}-\xi_{n}\right) \sum_{\nu=1}^{4} v_{\mu}^{(n)} v_{\nu}^{(n)}\left(A_{\nu}\right)_{l k}
$$

Let us comment on actual values we have chosen for the parameters in the potential (A.8). For the single-observable analysis in section 3, we typically ${ }^{18}$ used $\gamma_{n}=10^{4}$. For the multi-observable analysis in section 4, we studied the cases (a) and (b) in (4.10) corresponding to the $\mathrm{SO}(3)$ and $\mathrm{SO}(2)$ symmetric vacua, respectively. In the case (a), we set $\gamma_{1}=\gamma_{2}=0$ since $\lambda_{1}$ and $\lambda_{2}$ are automatically close to $\lambda_{3}$. When we fix $\lambda_{4}$ to the value obtained by GEM and vary $\lambda_{3}$ as we do in section 4.2 , we set $\gamma_{4}$ much larger than $\gamma_{3}$ (typically $\gamma_{4}=10^{5}$ and $\gamma_{3}=10^{2}$ ), and set $\xi_{4}$ to the GEM value for $\left\langle\lambda_{4}\right\rangle$ obtained with the $\mathrm{SO}(3)$ ansatz. In the case (b), we set $\gamma_{1}=0$ since $\lambda_{1}$ is automatically close to $\lambda_{2}$. When we fix $\lambda_{3}$ and $\lambda_{4}$ to the values obtained by GEM and vary $\lambda_{2}$ as we do in section 4.3, we set $\gamma_{3}$ and $\gamma_{4}$ much larger than $\gamma_{2}$ (typically $\gamma_{3}=\gamma_{4}=10^{5}$ and $\gamma_{2}=10^{2}$ ), and set $\xi_{3}$ and $\xi_{4}$ to the GEM values for $\left\langle\lambda_{3}\right\rangle$ and $\left\langle\lambda_{4}\right\rangle$, respectively, obtained with the $\mathrm{SO}(2)$ ansatz.

\footnotetext{
${ }^{18}$ In fact we have used $\gamma_{n}$ in the range $10^{3}-10^{7}$. We have checked that our results are independent of $\gamma_{n}$ within a wide range.
} 


\section{B. Asymptotic behaviors of the functions $f_{n}^{(0)}(x)$}

In this section we discuss the asymptotic behaviors of the functions $f_{n}^{(0)}(x)$ that appears in (3.17). While these behaviors are not explicitly used in our analysis, they help us understand the dynamical properties of the eigenvalues $\tilde{\lambda}_{n}$ in the matrix model. In fact the functions $f_{n}^{(0)}(x)$ in the IIB matrix model behaves quite differently as we mention in section 5, and we hope that our results given below would be useful for comparison.

Let us recall that the function $f_{n}^{(0)}(x)$ is defined by eq. (3.9) in terms of $\rho_{n}^{(0)}(x)$, which is the distribution function of $\tilde{\lambda}_{n}$ in the phase-quenched model. It is therefore expected to have simple scaling behavior when $x \ll 1$ or $x \gg 1$. At small $x$ we expect

$$
\frac{1}{N^{2}} f_{n}^{(0)}(x) \simeq\left\{\frac{1}{2}(5-n)+r \delta_{n 1}\right\} \frac{1}{x}+a_{n}
$$

This behavior is due to the phase space suppression since $(5-n)$ directions shrink as we decrease $x$. Considering that the shrunken directions have the extent proportional to $\sqrt{x}$, we obtain $\rho_{n}^{(0)}(x) \sim(\sqrt{x})^{(5-n) N^{2}}$. The $n=1$ case differs since all the eigenvalues of $A_{\mu}$ collapse to 0 at $x \ll 1$ and the suppression factor comes also from the fermion determinant, which is a homogeneous polynomial of $A_{\mu}$ of degree $2 r N^{2}$. This gives an extra suppression factor of $(\sqrt{x})^{2 r N^{2}}$. From the definition (3.9), we obtain the leading behavior of (B.1). Assuming that we have some analytic function of $x$ multiplied to the leading power-law behavior of $\rho_{n}^{(0)}(x)$, we obtain some constant $a_{n}$ in (B.1) as the subleading term.
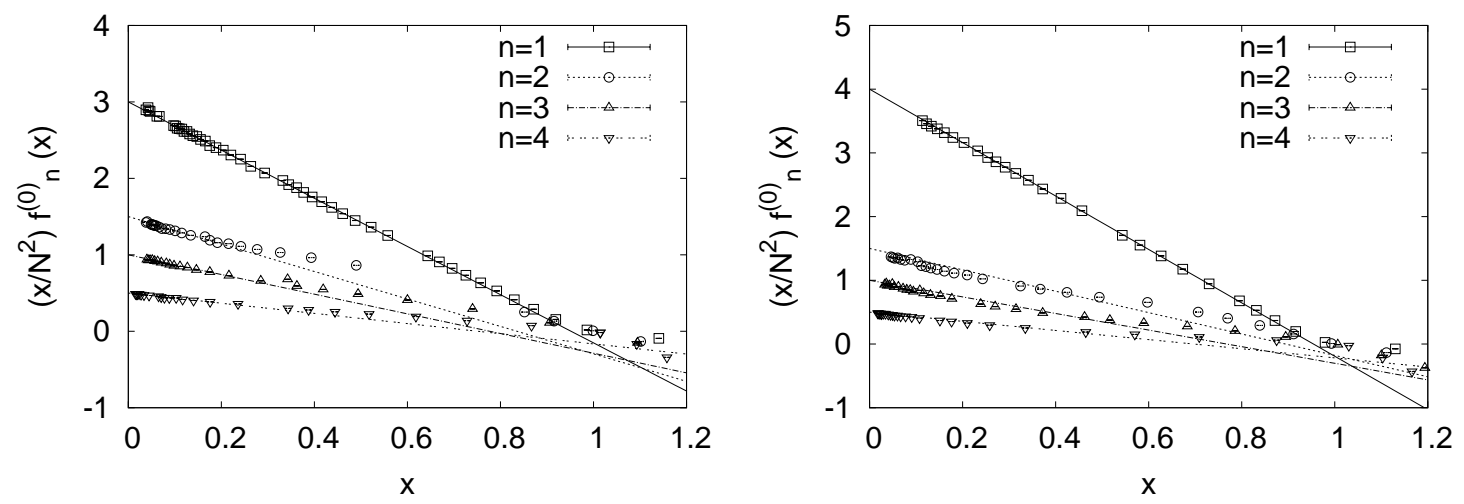

Figure 10: The function $\frac{x}{N^{2}} f_{n}^{(0)}(x)$ at $N=64$ is plotted against $x$ for $r=1$ (Left) and $r=2$ (Right). The straight lines are fits to the behavior (B.1).

At large $x$ we expect

$$
\frac{1}{N^{2}} f_{n}^{(0)}(x) \simeq-\frac{1}{2} n\left\langle\lambda_{n}\right\rangle_{0}+\left(\frac{n}{2}+r\right) \frac{1}{x}
$$

As we increase $x$, the eigenvalues $\lambda_{1}, \cdots, \lambda_{n}$ are forced to be large and the $(n+1)$-th through the 4 -th directions remain relatively small. In that case it is the bosonic action 

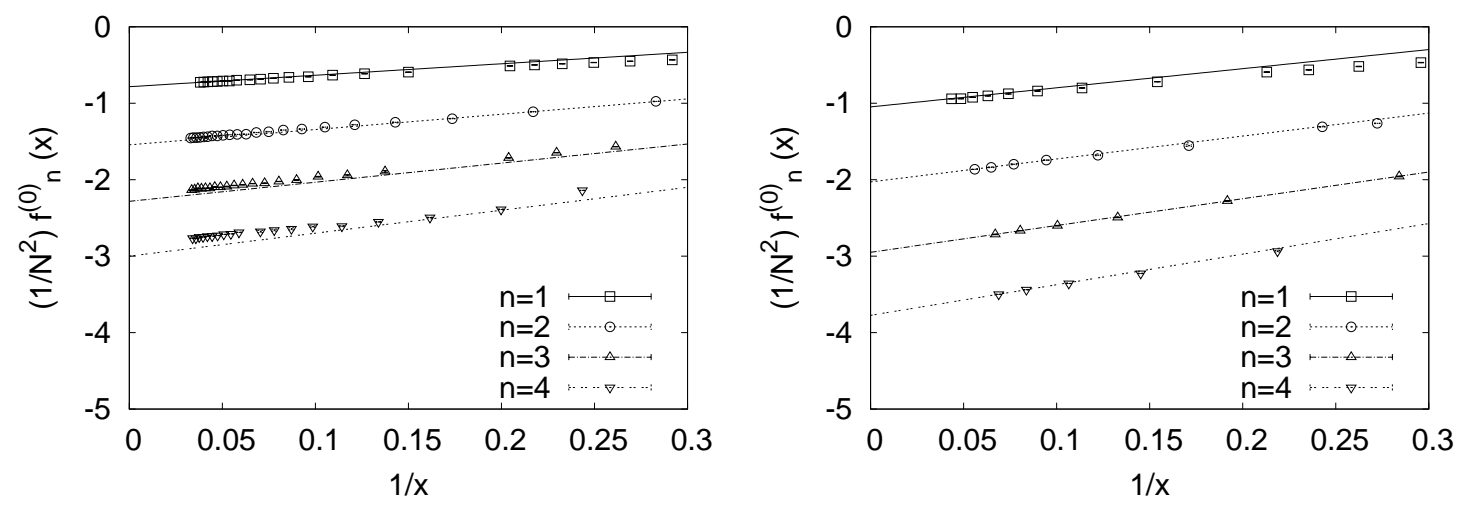

Figure 11: The function $\frac{1}{N^{2}} f_{n}^{(0)}(x)$ at $N=64$ is plotted against $1 / x$ for $r=1$ (Left) and $r=2$ (Right). The straight lines represent the behavior (B.2).

that dominates the suppression of those configurations and we expect that

$$
\rho_{n}^{(0)}(x) \sim \exp \left(-\left\langle\frac{1}{2} N \operatorname{tr}\left(A_{\mu}\right)^{2}\right\rangle_{n, V}\right) \sim \exp \left(-\frac{1}{2} N^{2} \sum_{k=1}^{n}\left\langle\lambda_{k}\right\rangle_{n, V}\right) .
$$

Since $\left\langle\lambda_{k}\right\rangle_{n, V} \sim x\left\langle\lambda_{n}\right\rangle_{0}$ at large $x$ for $k=1, \cdots, n$, we obtain the first term of (B.2), which becomes $-\frac{1}{2} n\left(1+\frac{r}{2}\right)$ at large $N$. We also expect a power-law correction $(\sqrt{x})^{(n+2 r) N^{2}}$ to $\rho_{n}^{(0)}(x)$ that comes from the measure and the fermion determinant as we discussed in the case of $x \ll 1$. Thus we obtain the second term in (B.2) as the subleading term. Figures 10 and 11 confirm the above asymptotic behaviors at small $x$ and large $x$, respectively.

\section{Large- $N$ extrapolations in the single-observable analysis}

In this section we explain the large- $N$ extrapolations made in the single-observable analysis based on the asymptotic behavior (3.18).

\begin{tabular}{|c|c|c|c|c|}
\hline & \multicolumn{2}{|c|}{$r=1$} & \multicolumn{2}{c|}{$r=2$} \\
\hline$n$ & $c_{n}$ & $d_{n}$ & $c_{n}$ & $d_{n}$ \\
\hline 1 & & $2.26(6)$ & & $2.02(5)$ \\
2 & $2.3(1)$ & $0.378(2)$ & $2.407(2)$ & $0.53(2)$ \\
3 & $0.52(4)$ & $0.20(3)$ & $0.96(6)$ & $0.353(3)$ \\
4 & $0.26(4)$ & & $0.46(8)$ & \\
\hline
\end{tabular}

Table 4: Large- $N$ extrapolated values of the coefficients $c_{n}$ and $d_{n}$ in the asymptotic behaviors (3.18).
In figs. 12 and 13 we show the log$\log$ plots of $-\frac{1}{N^{2}} \log w_{n}(x)$ for $r=1$ and $r=2$, respectively. We fit the data to the behavior (3.18) at $x \ll 1$ and at $x \gg 1$, and extract the coefficients $c_{n}$ and $d_{n}$ in (3.18) for each $N$. The results are plotted against $\frac{1}{N}$ in figs. 14 and 15 for $r=1$ and $r=2$, respectively. Assuming that the finite- $N$ effects are $\mathrm{O}(1 / N)$, we make large- $N$ extrapolations, which give the values shown in Table 4. The corresponding

functions (3.18), which we define as the scaling functions $\Phi_{n}(x)$ in each region, are also plotted in figs. 12 and 13. (The two solid lines show the margin of error.) The scaling functions thus obtained are plotted in the left column of figs. 2 and 3. 

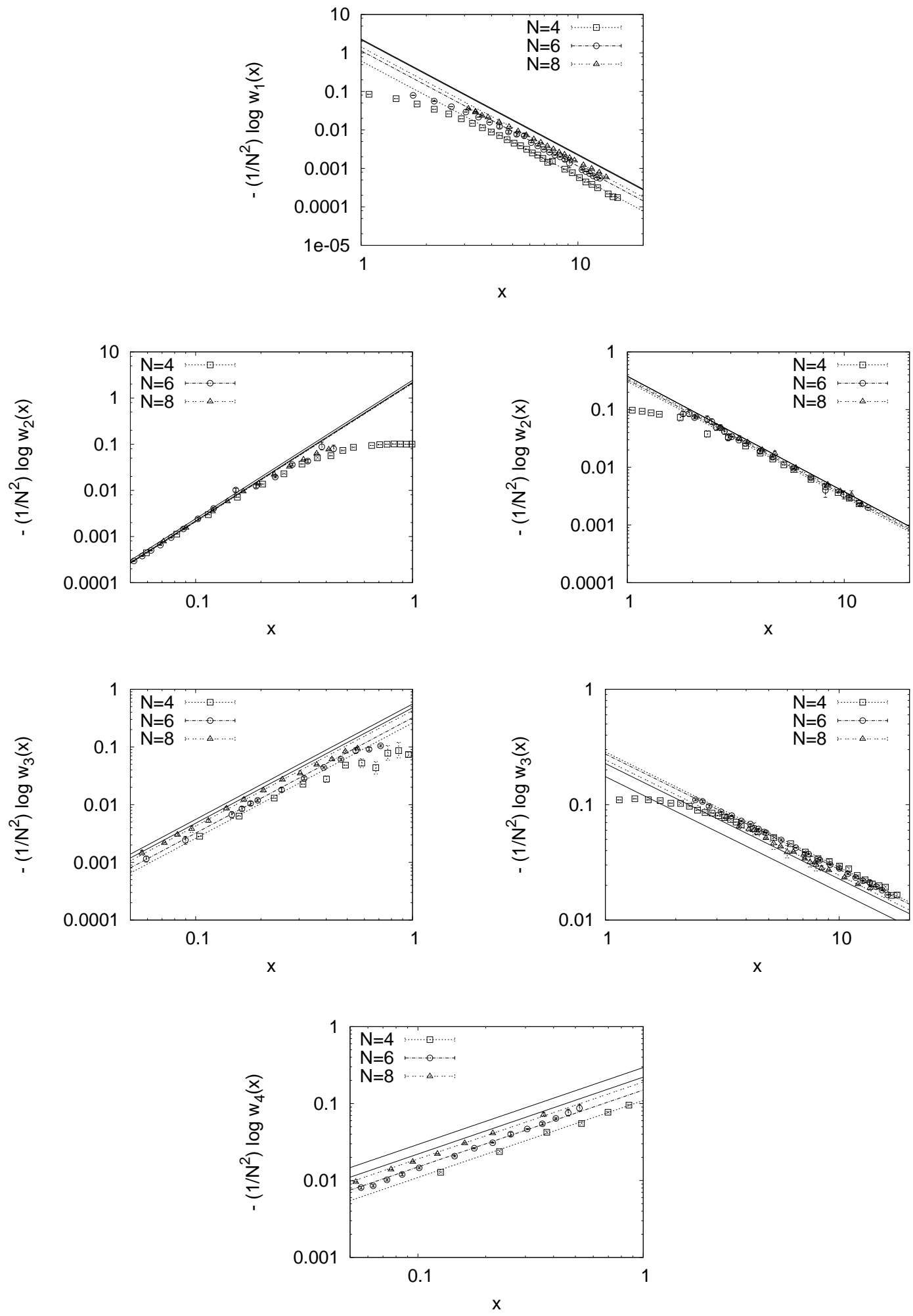

Figure 12: The $\log -\log$ plot of $-\frac{1}{N^{2}} \log w_{n}(x)$ for $r=1$. The straight solid lines represent the power-law behavior (3.18) with the coefficients presented in Table 4, which are obtained by the large- $N$ extrapolation from the $N=4,6,8$ data as described in fig. 14. A clear trend towards large- $N$ scaling is observed, in particular, for the $n=2,3$ cases. 

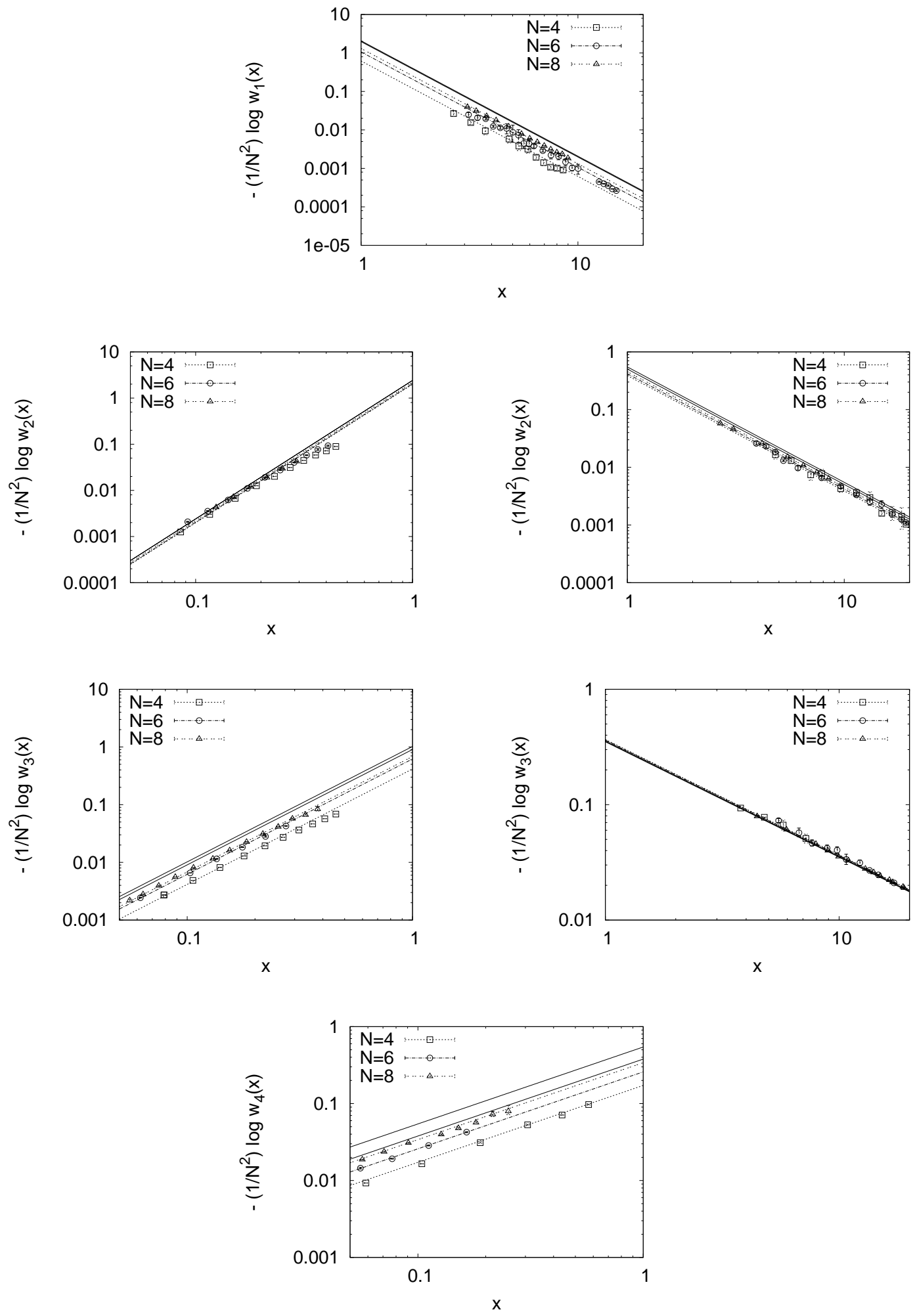

Figure 13: The $\log -\log$ plot of $-\frac{1}{N^{2}} \log w_{n}(x)$ for $r=2$. The straight solid lines represent the power-law behavior (3.18) with the coefficients presented in Table 4, which are obtained by the large- $N$ extrapolation from the $N=4,6,8$ data as described in fig. 15. A clear trend towards large- $N$ scaling is observed, in particular, for the $n=2,3$ cases. 

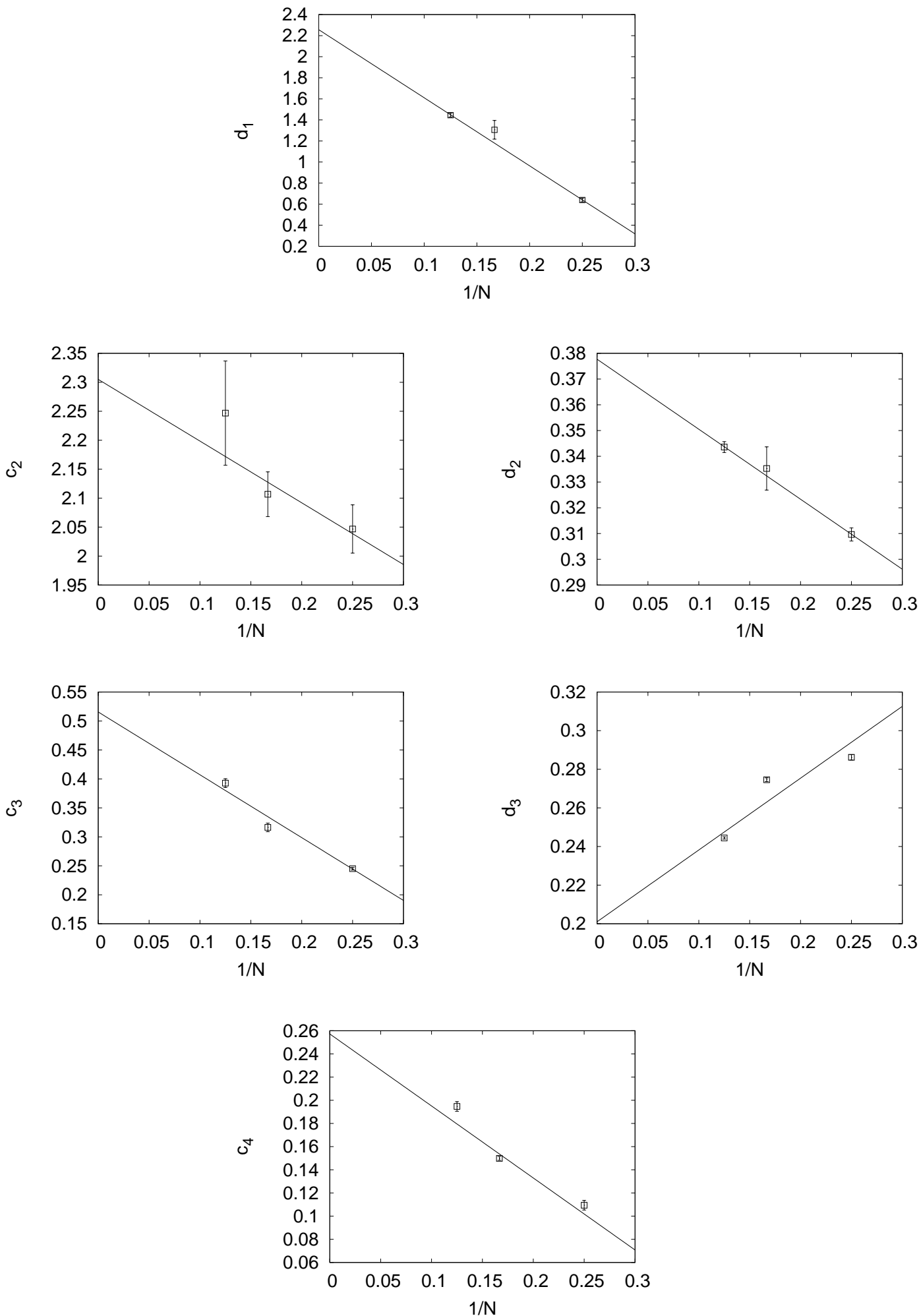

Figure 14: The coefficients $c_{n}$ and $d_{n}$ in the asymptotic formula (3.18) for $r=1$ extracted from fig. 12 are plotted against $\frac{1}{N}$. The data points are fitted to a straight line. The large- $N$ extrapolated values obtained in this way are presented in Table 4. 

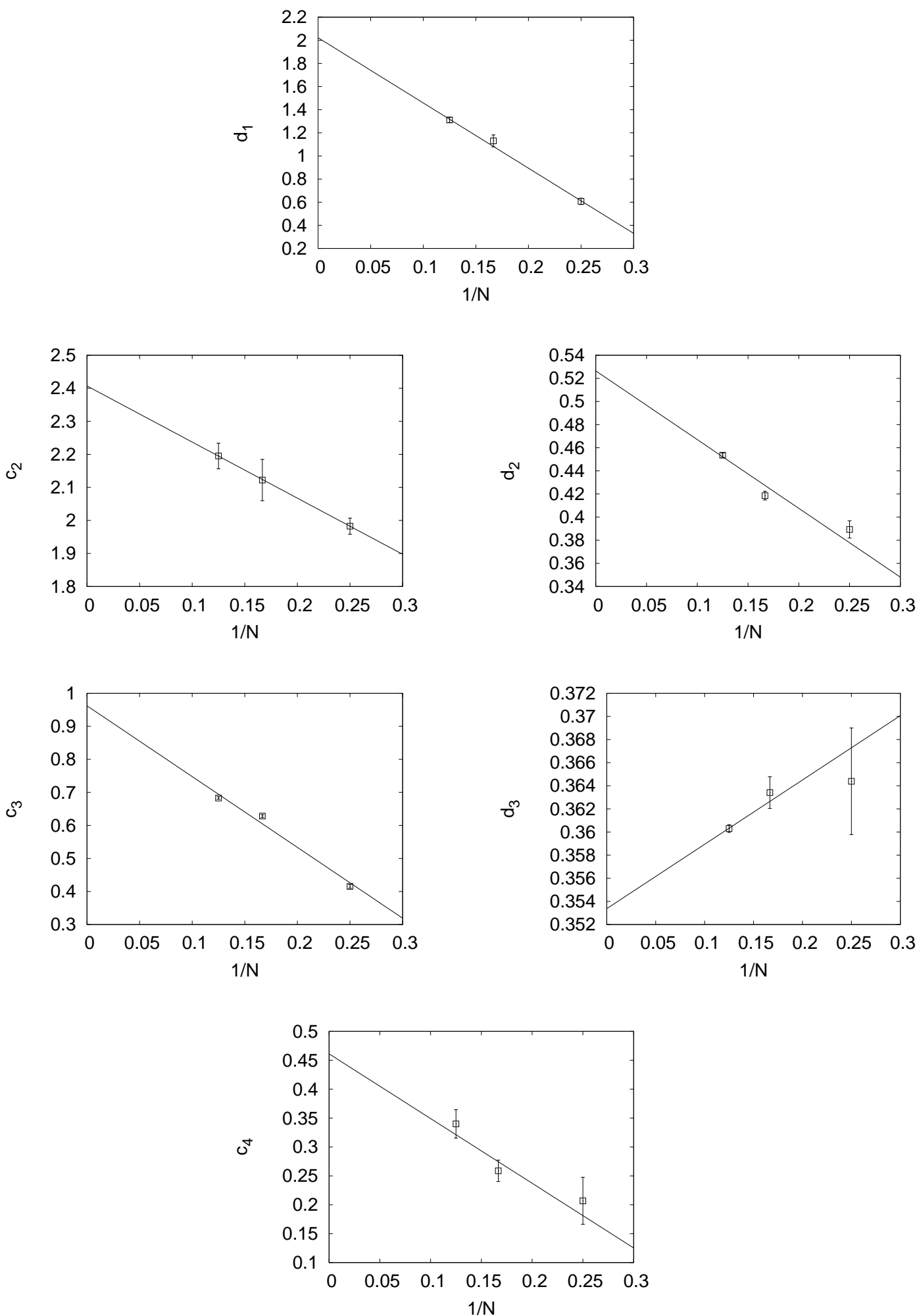

Figure 15: The coefficients $c_{n}$ and $d_{n}$ in the asymptotic formula (3.18) for $r=2$ extracted from fig. 13 are plotted against $\frac{1}{N}$. The data points are fitted to a straight line. The large- $N$ extrapolated values obtained in this way are presented in Table 4. 


\section{Large- $N$ extrapolations in the multi-observable analysis}

In this section we summarize the large- $N$ extrapolations we made in the multi-observable case. We describe them for the $\mathrm{SO}(3)$ and $\mathrm{SO}(2)$ symmetric vacua separately.

\section{D.1 extrapolations for the $\mathrm{SO}(3)$ symmetric vacuum}

Similarly to the asymptotic behavior (3.18) used in the single-observable analysis, it is expected that

$$
\frac{1}{N^{2}} \log w_{\mathrm{SO}(3)}(x, 0.5) \sim-\tilde{d}_{1} x^{-1}+\tilde{d}_{2} x^{-3 / 2}
$$

at $x \gg 1$. Here we have added the subleading term motivated from the fact that $\delta A /|A| \propto$ $1 / \sqrt{x}$ as described below (3.18).

In fig. 16 (Top), we plot $\frac{x}{N^{2}} \log w_{\mathrm{SO}(3)}(x, 0.5)$ against $\frac{1}{\sqrt{x}}$, which confirms (D.1) including the subleading term. We plot the coefficients $\tilde{d}_{1}$ and $\tilde{d}_{2}$ against $\frac{1}{N}$ in fig. 16 (Bottom), which shows that the finite $N$ effects are of the order of $1 / N$. Based on this observation, we obtain the large- $N$ extrapolated values $\tilde{d}_{1}=0.1572(5)$ and $\tilde{d}_{2}=-0.032(4)$. The scaling

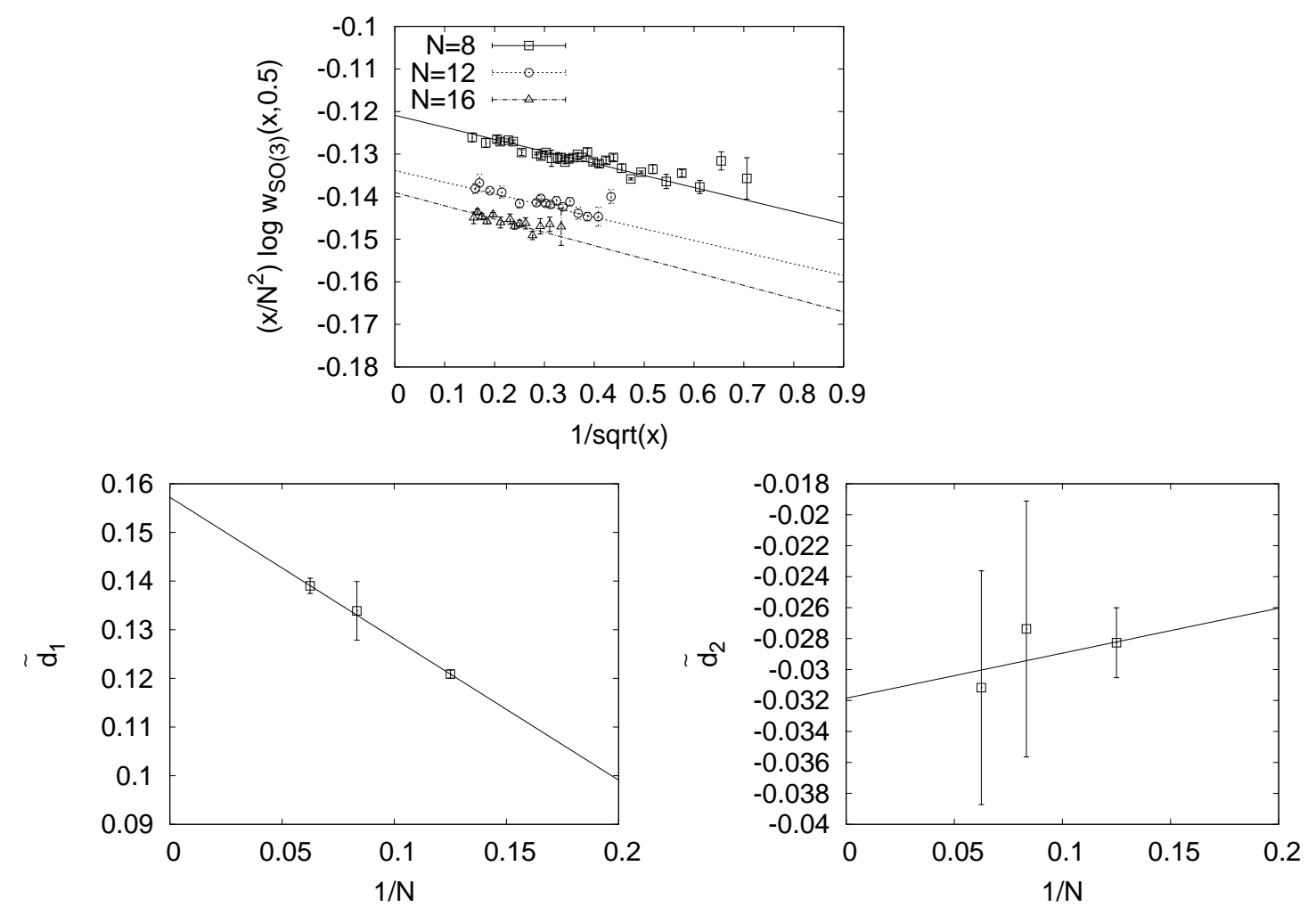

Figure 16: (Top) The function $\frac{x}{N^{2}} \log w_{\mathrm{SO}(3)}(x, 0.5)$ is plotted against $\frac{1}{\sqrt{x}}$ for $N=8,12,16$. The straight lines represent the fits to the asymptotic behavior (D.1). (Bottom) The coefficients $\tilde{d}_{1}$ and $\tilde{d}_{2}$ in the asymptotic behavior (D.1) extracted from the top figure are plotted against $\frac{1}{N}$. The data points can be fitted nicely to a straight line. 
function (4.15) obtained in this way is plotted in fig. 4 (Left). By plugging $x=1.17$ into (D.1), we obtain $\Phi_{\mathrm{SO}(3)}(1.17,0.5)=-0.160(3)$ as in eq. (4.32).

We redo the calculation for $y=0.45,0.55$ and obtain the large- $N$ extrapolated values

$$
\begin{array}{ll}
\tilde{d}_{1}=0.1433(2), & \tilde{d}_{2}=-0.023(1) \quad \text { for } y=0.45 \\
\tilde{d}_{1}=0.1749(2), & \tilde{d}_{2}=-0.029(3) \quad \text { for } y=0.55
\end{array}
$$

from which we obtain $\Phi_{\mathrm{SO}(3)}(1.17, y)$ for $y=0.45$ and 0.55 . These results, together with the value at $y=0.5$, are plotted in fig. 5 (Left).

\section{D.2 extrapolations for the $\mathrm{SO}(2)$ symmetric vacuum}

Similarly to (D.1) for the $\mathrm{SO}(3)$ symmetric vacuum, it is expected that

$$
\frac{1}{N^{2}} \log w_{\mathrm{SO}(2)}(x, 0.7,0.5) \simeq-\tilde{d}_{1} x^{-2}+\tilde{d}_{2} x^{-5 / 2}
$$

at $x \gg 1$. In fig. 17 (Top), we plot $\frac{x^{2}}{N^{2}} \log w_{\mathrm{SO}(2)}(x, 0.7,0.5)$ against $\frac{1}{\sqrt{x}}$, which confirms (D.4) including the subleading term. We extract the coefficients $\tilde{d}_{1}$ and $\tilde{d}_{2}$ for $N=8,12,16$,
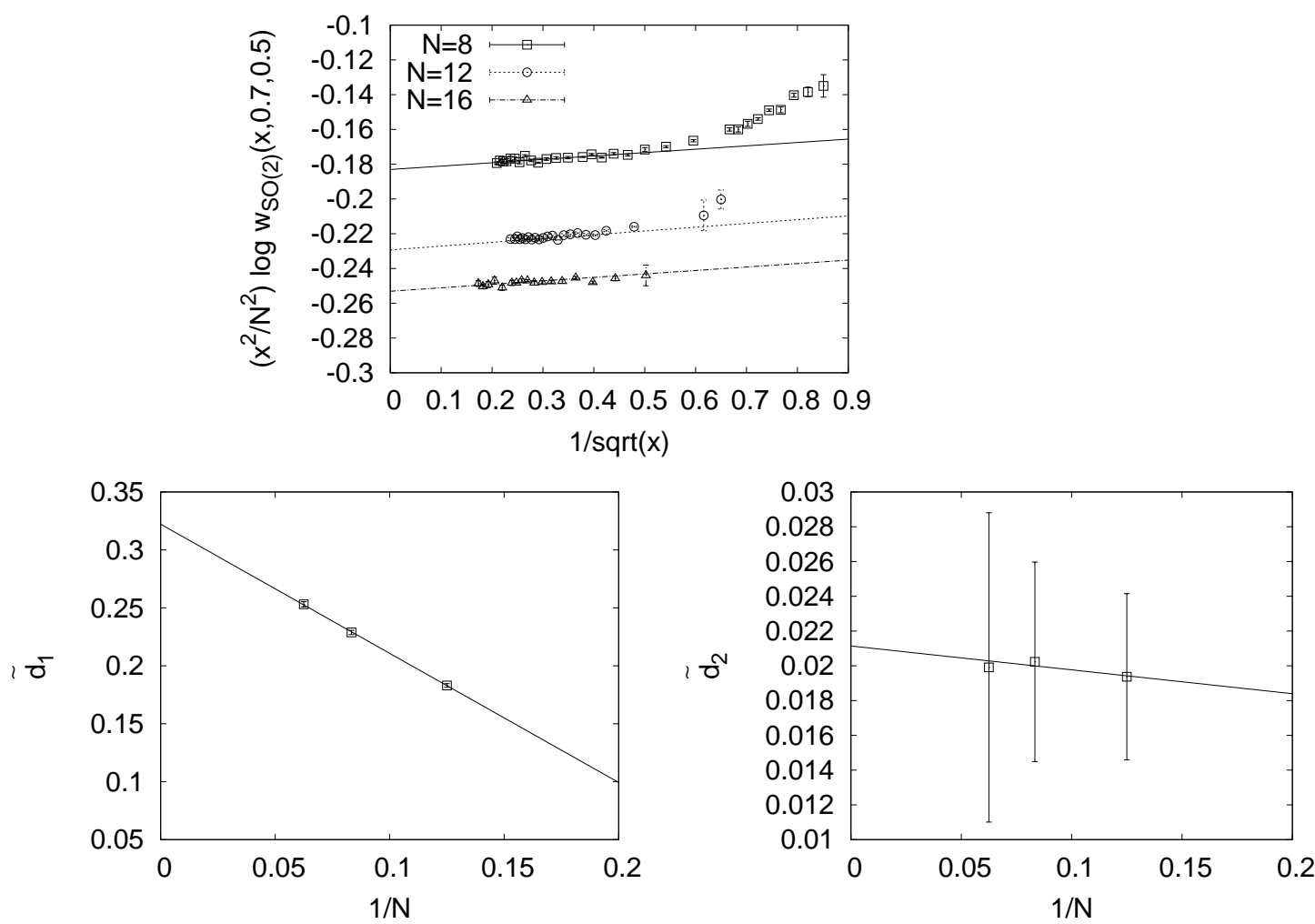

Figure 17: (Top) The function $\frac{x^{2}}{N^{2}} \log w_{\mathrm{SO}(2)}(x, 0.7,0.5)$ is plotted against $\frac{1}{\sqrt{x}}$ for $N=8,12,16$. The straight lines represent the fits to the asymptotic behavior (D.4). (Bottom) The coefficients $\tilde{d}_{1}$ and $\tilde{d}_{2}$ in the asymptotic behavior (D.4) extracted from the top figure are plotted against $\frac{1}{N}$. The data points can be fitted nicely to a straight line. 
and make a large- $N$ extrapolation for $\tilde{d}_{1}$ and $\tilde{d}_{2}$ as in fig. 17 (Bottom), from which we get the large- $N$ extrapolated values $\tilde{d}_{1}=0.322(2)$ and $\tilde{d}_{2}=0.021(1)$. The scaling function (4.20) obtained in this way is plotted in fig. 6 (Left). By plugging $x=1.4$ into (D.4), we obtain $\Phi_{\mathrm{SO}(2)}(1.4,0.7,0.5)=-0.155(1)$ as in eq. (4.33).

We redo the calculation for $y=0.65,0.75$, and obtain the large- $N$ extrapolated values

$$
\begin{array}{ll}
\tilde{d}_{1}=0.295(3), & \tilde{d}_{2}=0.018(1) \quad \text { for } y=0.65 \\
\tilde{d}_{1}=0.344(3), & \tilde{d}_{2}=0.019(3) \quad \text { for } y=0.75
\end{array}
$$

from which we obtain $\Phi_{\mathrm{SO}(2)}(1.4, y, 0.5)$ at $y=0.65,0.75$. These results, together with the value at $y=0.7$, are plotted in fig. 7 (Left).

We redo the calculation for $z=0.45,0.55$, and obtain the large- $N$ extrapolated values

$$
\begin{array}{ll}
\tilde{d}_{1}=0.292(5), \quad \tilde{d}_{2}=0.026(2) \quad \text { for } z=0.45 \\
\tilde{d}_{1}=0.352(2), \quad \tilde{d}_{2}=0.015(3) \quad \text { for } z=0.55
\end{array}
$$

from which we obtain $\Phi_{\mathrm{SO}(2)}(1.4,0.7, z)$ at $z=0.45,0.55$. These results, together with the value at $z=0.5$, are plotted in fig. 8 (Left).
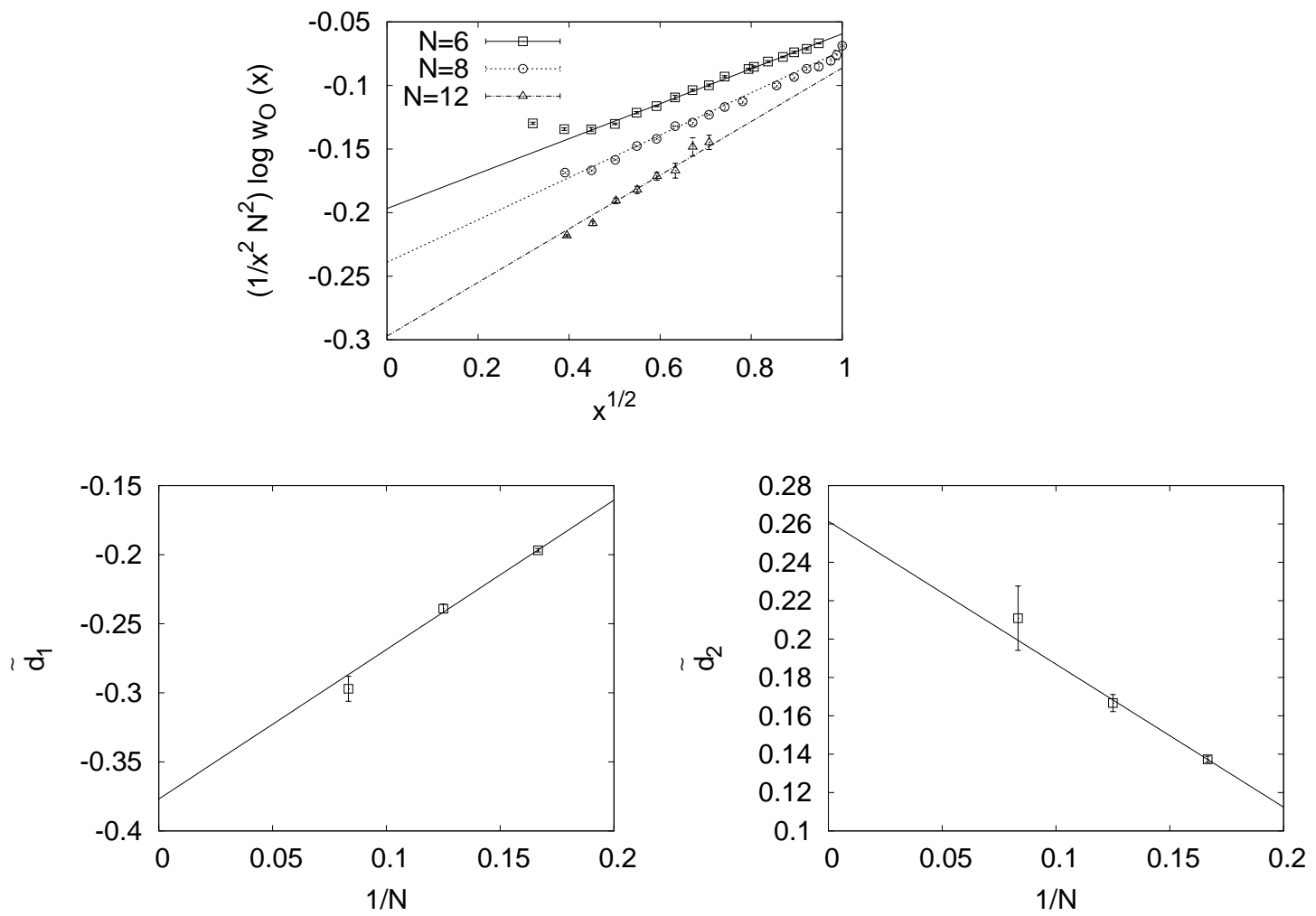

Figure 18: (Top) The function $\frac{1}{x^{2} N^{2}} \log w_{\mathcal{O}}(x)$ is plotted against $\sqrt{x}$ for $N=8,12,16$. (Bottom) The coefficients $\tilde{d}_{1}$ and $\tilde{d}_{2}$ in the asymptotic behavior (D.9) are plotted against $\frac{1}{N}$. The data points can be fitted nicely to a straight line. 
Finally let us describe the large- $N$ extrapolations used in section 4.7 in the analysis for the observable (4.40). First the asymptotic behavior of $\frac{1}{N^{2}} \log w_{\mathcal{O}}(x)$ is expected to be

$$
\frac{1}{N^{2}} \log w_{\mathcal{O}}(x)=-\tilde{d}_{1} x^{2}+\tilde{d}_{2} x^{5 / 2}
$$

The leading term is obtained as follows. Let us consider a small perturbation around a diagonal configuration. The phase of the fermion determinant appears at the second order of this perturbation. On the other hand, the observable $\mathcal{O}$ becomes nonzero also at the second order. Therefore the distribution of the phase is expected to have a width $\sigma \propto x$. Applying the formula (3.19), we obtain the leading term. The power of the subleading term can be deduced by expanding the fermion determinant around a diagonal configuration.

In fig. 18 (Top) we plot $\frac{1}{x^{2} N^{2}} \log w_{\mathcal{O}}(x)$ against $\sqrt{x}$. The data points can be fitted nicely by straight lines, which confirms the asymptotic behavior (D.9). We extract the

coefficients $\tilde{d}_{1}$ and $\tilde{d}_{2}$ for $N=6,8,12$, and make a large- $N$ extrapolation for $\tilde{d}_{1}$ and $\tilde{d}_{2}$ as in fig. 18 (Bottom). We obtain $\tilde{d}_{1}=0.38(2)$ and $\tilde{d}_{2}=0.26(1)$. The scaling function (4.46) obtained in this way is plotted in fig. 9 (Left).

\section{References}

[1] J. M. Maldacena, The large $N$ limit of superconformal field theories and supergravity, Adv. Theor. Math. Phys. 2 (1998) 231 [Int. J. Theor. Phys. 38 (1999) 1113] [hep-th/9711200].

[2] M. Hanada, J. Nishimura and S. Takeuchi, Non-lattice simulation for supersymmetric gauge theories in one dimension, Phys. Rev. Lett. 99 (2007) 161602 [arXiv:0706.1647].

[3] S. Catterall and T. Wiseman, Towards lattice simulation of the gauge theory duals to black holes and hot strings, JHEP 0712 (2007) 104 [arXiv:0706.3518].

[4] K. N. Anagnostopoulos, M. Hanada, J. Nishimura and S. Takeuchi, Monte Carlo studies of supersymmetric matrix quantum mechanics with sixteen supercharges at finite temperature, Phys. Rev. Lett. 100 (2008) 021601 [arXiv:0707.4454].

[5] S. Catterall and T. Wiseman, Black hole thermodynamics from simulations of lattice Yang-Mills theory, Phys. Rev. D 78 (2008) 041502 [arXiv:0803.4273].

[6] M. Hanada, Y. Hyakutake, J. Nishimura and S. Takeuchi, Higher derivative corrections to black hole thermodynamics from supersymmetric matrix quantum mechanics, Phys. Rev. Lett. 102 (2009) 191602 [arXiv:0811.3102].

[7] S. Catterall and T. Wiseman, Extracting black hole physics from the lattice, JHEP 1004 (2010) 077 [arXiv:0909.4947].

[8] M. Hanada, A. Miwa, J. Nishimura and S. Takeuchi, Schwarzschild radius from Monte Carlo calculation of the Wilson loop in supersymmetric matrix quantum mechanics, Phys. Rev. Lett. 102 (2009) 181602 [arXiv:0811.2081].

[9] M. Hanada, J. Nishimura, Y. Sekino and T. Yoneya, Monte Carlo studies of Matrix theory correlation functions, Phys. Rev. Lett. 104 (2010) 151601 [arXiv:0911.1623];

Direct test of the gauge-gravity correspondence for Matrix theory correlation functions, arXiv:1108.5153. 
[10] J. Nishimura, Non-lattice simulation of supersymmetric gauge theories as a probe to quantum black holes and strings, PoS LAT2009 (2009) 016 [arXiv:0912.0327].

[11] N. Itzhaki, J. M. Maldacena, J. Sonnenschein and S. Yankielowicz, Supergravity and the large $N$ limit of theories with sixteen supercharges, Phys. Rev. D 58 (1998) 046004 [hep-th/9802042].

[12] N. Ishibashi, H. Kawai, Y. Kitazawa and A. Tsuchiya, A large- $N$ reduced model as superstring, Nucl. Phys. B 498 (1997) 467 [hep-th/9612115];

for a comprehensive review see e.g. H. Aoki, S. Iso, H. Kawai, Y. Kitazawa, A. Tsuchiya and T. Tada, IIB matrix model, Prog. Theor. Phys. Suppl. 134 (1999) 47 [hep-th/9908038];

T. Azuma, Matrix models and the gravitational interaction, hep-th/0401120.

[13] H. Aoki, S. Iso, H. Kawai, Y. Kitazawa and T. Tada, Space-time structures from IIB matrix model, Prog. Theor. Phys. 99 (1998) 713 [hep-th/9802085].

[14] V. A. Kostelecky and S. Samuel, Spontaneous breaking of Lorentz symmetry in string theory, Phys. Rev. D 39 (1989) 683.

[15] J. Nishimura and F. Sugino, Dynamical generation of four-dimensional space-time in the IIB matrix model, JHEP 0205 (2002) 001 [hep-th/0111102].

[16] H. Kawai, S. Kawamoto, T. Kuroki, T. Matsuo and S. Shinohara, Mean field approximation of IIB matrix model and emergence of four dimensional space-time, Nucl. Phys. B 647 (2002) 153 [hep-th/0204240];

H. Kawai, S. Kawamoto, T. Kuroki and S. Shinohara, Improved perturbation theory and four-dimensional space-time in IIB matrix model, Prog. Theor. Phys. 109 (2003) 115 [hep-th/0211272].

T. Aoyama and H. Kawai, Higher order terms of improved mean field approximation for IIB matrix model and emergence of four-dimensional space-time, Prog. Theor. Phys. 116 (2006) 405 [hep-th/0603146].

[17] J. Nishimura, T. Okubo and F. Sugino, Systematic study of the SO(10) symmetry breaking vacua in the matrix model for type IIB superstrings, arXiv:1108.1293.

[18] S.-W. Kim, J. Nishimura and A. Tsuchiya, Expanding (3+1)-dimensional universe from a Lorentzian matrix model for superstring theory in (9+1)-dimensions, arXiv:1108.1540.

[19] W. Krauth, H. Nicolai and M. Staudacher, Monte Carlo approach to M-theory, Phys. Lett. B 431 (1998) 31 [hep-th/9803117];

W. Krauth and M. Staudacher, Finite Yang-Mills integrals, Phys. Lett. B 435 (1998) 350 [hep-th/9804199];

W. Krauth and M. Staudacher, Eigenvalue distributions in Yang-Mills integrals, Phys. Lett. $B 453$ (1999) 253 [hep-th/9902113].

[20] T. Hotta, J. Nishimura and A. Tsuchiya, Dynamical aspects of large $N$ reduced models, Nucl. Phys. B 545 (1999) 543 [hep-th/9811220].

[21] J. Ambjorn, K. N. Anagnostopoulos, W. Bietenholz, T. Hotta and J. Nishimura, Monte Carlo studies of the IIB matrix model at large N, JHEP 0007 (2000) 011 [hep-th/0005147].

[22] J. Ambjorn, K. N. Anagnostopoulos, W. Bietenholz, T. Hotta and J. Nishimura, Large N dynamics of dimensionally reduced $4 D S U(N)$ super Yang-Mills theory, JHEP 0007 (2000) 013 [hep-th/0003208]; 
J. Ambjorn, K. N. Anagnostopoulos, W. Bietenholz, F. Hofheinz and J. Nishimura, On the spontaneous breakdown of Lorentz symmetry in matrix models of superstrings, Phys. Rev. D 65 (2002) 086001 [hep-th/0104260].

[23] P. Bialas, Z. Burda, B. Petersson and J. Tabaczek, Large N limit of the IKKT matrix model, Nucl. Phys. B 592 (2001) 391 [hep-lat/0007013];

Z. Burda, B. Petersson and J. Tabaczek, Geometry of reduced supersymmetric $4 D$ Yang-Mills integrals, Nucl. Phys. B 602 (2001) 399 [hep-lat/0012001];

Z. Burda, B. Petersson and M. Wattenberg, Semiclassical geometry of $4 D$ reduced supersymmetric Yang-Mills integrals, JHEP 0503 (2005) 058 [hep-th/0503032].

[24] K. N. Anagnostopoulos and J. Nishimura, New approach to the complex-action problem and its application to a nonperturbative study of superstring theory, Phys. Rev. D 66 (2002) 106008 [hep-th/0108041].

[25] J. Ambjorn, K. N. Anagnostopoulos, J. Nishimura and J. J. M. Verbaarschot, The factorization method for systems with a complex action: a test in Random Matrix Theory for finite density QCD, JHEP 0210 (2002) 062 [hep-lat/0208025];

J. Ambjorn, K. N. Anagnostopoulos, J. Nishimura and J. J. M. Verbaarschot, Non-commutativity of the zero chemical potential limit and the thermodynamic limit in finite density systems, Phys. Rev. D 70 (2004) 035010 [hep-lat/0402031].

[26] V. Azcoiti, G. Di Carlo, A. Galante and V. Laliena, New proposal for numerical simulations of $\theta$-vacuum like systems, Phys. Rev. Lett. 89 (2002) 141601 [hep-lat/0203017].

[27] Z. Fodor, S. D. Katz and C. Schmidt, The density of states method at non-zero chemical potential, JHEP 0703 (2007) 121 [hep-lat/0701022].

Z. Fodor and S. D. Katz, A new method to study lattice $Q C D$ at finite temperature and chemical potential, Phys. Lett. B 534 (2002) 87 [hep-lat/0104001].

Z. Fodor and S. D. Katz, Critical point of $Q C D$ at finite $T$ and mu, lattice results for physical quark masses, JHEP 0404 (2004) 050 [hep-lat/0402006].

[28] S. Ejiri, On the existence of the critical point in finite density lattice QCD, Phys. Rev. D $\mathbf{7 7}$ (2008) 014508 [arXiv:0706.3549].

[29] M. P. Lombardo, K. Splittorff and J. J. M. Verbaarschot, Distributions of the phase angle of the fermion determinant in QCD, Phys. Rev. D 80 (2009) 054509 [arXiv:0904.2122];

M. P. Lombardo, K. Splittorff and J. J. M. Verbaarschot, The fluctuations of the quark number and of the chiral condensate, Phys. Rev. D 81 (2010) 045012 [arXiv:0910.5482].

[30] W. Unger, P. de Forcrand, Continuous time Monte Carlo for lattice QCD in the strong coupling limit," arXiv:1107.1553;

P. de Forcrand, O. Philipsen, Constraining the QCD phase diagram by tricritical lines at imaginary chemical potential, Phys. Rev. Lett. 105 (2010) 152001 [arXiv:1004.3144].

J. Bloch, Evading the sign problem in random matrix simulations, arXiv:1103.3467;

J. Bloch, T. Wettig, The QCD sign problem and dynamical simulations of random matrices, JHEP 05 (2011) 048 [arXiv: 1102.3715];

G. Aarts, F. A. James, E. Seiler, I. -O. Stamatescu, Complex Langevin: Etiology and diagnostics of its main problem, arXiv:1101.3270;

G. Aarts, F. A. James, On the convergence of complex Langevin dynamics: The three-dimensional XY model at finite chemical potential, JHEP 08 (2010) 020 [arXiv: 1005.3468]; 
S. Chandrasekharan, A. Li, Fermion bag approach to the sign problem in strongly coupled lattice QED with Wilson fermions, JHEP 01 (2011) 018 [arXiv:1008.5146];

C. R. Allton, S. Ejiri, S. J. Hands, O. Kaczmarek, F. Karsch, E. Laermann, C. Schmidt, L. Scorzato, The QCD thermal phase transition in the presence of a small chemical potential, Phys. Rev. D 66 (2002) 074507 [hep-lat/0204010];

R. V. Gavai, S. Gupta, Pressure and nonlinear susceptibilities in QCD at finite chemical potentials, Phys. Rev. D 68 (2003) 034506 [hep-lat/0303013];

M. D'Elia, M. -P. Lombardo, Finite density QCD via imaginary chemical potential, Phys. Rev. D 67 (2003) 014505 [hep-lat/0209146];

P. de Forcrand, O. Philipsen, The QCD phase diagram for small densities from imaginary chemical potential, Nucl. Phys. B 642 (2002) 290 [hep-lat/0205016];

W. Bietenholz, A. Pochinsky, U. J. Wiese, Meron cluster simulation of the theta vacuum in the 2-d O(3) model, Phys. Rev. Lett. 75 (1995) 4524 [hep-lat/9505019].

[31] M. Creutz, Microcanonical Monte Carlo simulation, Phys. Rev. Lett. 50 (1983) 1411;

G. Bhanot, K. Bitar and R. Salvador, On solving four-dimensional SU(2) gauge theory by numerically finding its partition function, Phys. Lett. B 188 (1987) 246;

G. Bhanot, A. Gocksch and P. Rossi, On simulating complex actions, Phys. Lett. B 199 (1987) 101;

A. Gocksch, Simulating lattice QCD at finite density, Phys. Rev. Lett. 61 (1988) 2054;

M. Karliner, S. R. Sharpe and Y. F. Chang, Zeroing in on SU(3), Nucl. Phys. B 302 (1988) 204 ;

A. Gocksch, The Riemann walk: A method for simulating complex actions, Phys. Lett. B 206 (1988) 290.

[32] J. Nishimura and G. Vernizzi, Spontaneous breakdown of Lorentz invariance in IIB matrix model, JHEP 0004 (2000) 015 [hep-th/0003223];

J. Nishimura and G. Vernizzi, Brane world from IIB matrices, Phys. Rev. Lett. 85 (2000) 4664 [hep-th/0007022].

[33] G. Vernizzi and J. F. Wheater, Rotational symmetry breaking in multi-matrix models, Phys. Rev. D 66 (2002) 085024 [Erratum-ibid. D 67 (2003) 029904] [hep-th/0206226].

[34] J. Nishimura, Exactly solvable matrix models for the dynamical generation of space-time in superstring theory, Phys. Rev. D 65 (2002) 105012 [hep-th/0108070].

[35] J. Nishimura, T. Okubo and F. Sugino, Gaussian expansion analysis of a matrix model with the spontaneous breakdown of rotational symmetry, Prog. Theor. Phys. 114 (2005) 487 [hep-th/0412194].

[36] K. N. Anagnostopoulos, T. Azuma and J. Nishimura, A general approach to the sign problem: the factorization method with multiple observables, Phys. Rev. D 83 (2011) 054504 [arXiv: 1009.4504].

[37] K. N. Anagnostopoulos, T. Azuma and J. Nishimura, work in progress.

[38] T. Aoyama, J. Nishimura and T. Okubo, Spontaneous breaking of the rotational symmetry in dimensionally reduced super Yang-Mills models, Prog. Theor. Phys. 125 (2011) 537 [arXiv: 1007.0883].

[39] K. N. Anagnostopoulos, T. Azuma, K. Nagao and J. Nishimura, Impact of supersymmetry on the nonperturbative dynamics of fuzzy spheres, JHEP 0509 (2005) 046 [hep-th/0506062]. 